\title{
VMS-ROT: A New Module of the Virtual Multifrequency Spectrometer for Simulation, Interpretation, and Fitting of Rotational Spectra
}

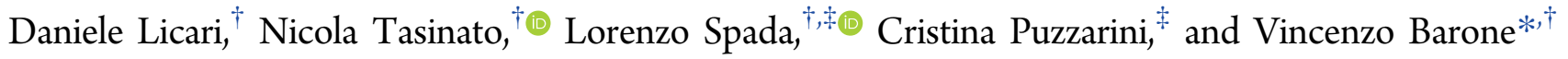 \\ ${ }^{\dagger}$ Scuola Normale Superiore, Piazza dei Cavalieri 7, I-56126 Pisa, Italy \\ "Dipartimento di Chimica “Giacomo Ciamician”, Università di Bologna, Via Selmi 2, I-40126 Bologna, Italy
}

ABSTRACT: The Virtual Multifrequency Spectrometer (VMS) is a tool that aims at integrating a wide range of computational and experimental spectroscopic techniques with the final goal of disclosing the static and dynamic physicalchemical properties "hidden" in molecular spectra. VMS is composed of two parts, namely, VMS-Comp, which provides access to the latest developments in the field of computational spectroscopy, and VMS-Draw, which provides a powerful graphical user interface (GUI) for an intuitive interpretation of theoretical outcomes and a direct comparison to experiment.

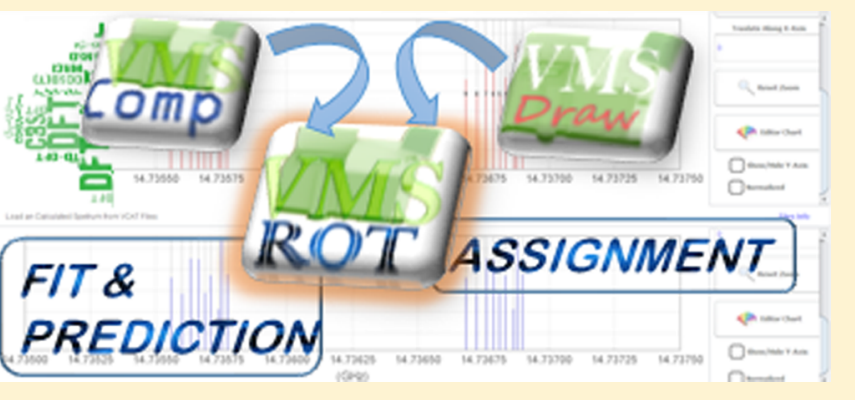
In the present work, we introduce VMS-ROT, a new module of VMS that has been specifically designed to deal with rotational spectroscopy. This module offers an integrated environment for the analysis of rotational spectra: from the assignment of spectral transitions to the refinement of spectroscopic parameters and the simulation of the spectrum. While bridging theoretical and experimental rotational spectroscopy, VMS-ROT is strongly integrated with quantum-chemical calculations, and it is composed of four independent, yet interacting units: (1) the computational engine for the calculation of the spectroscopic parameters that are employed as a starting point for guiding experiments and for the spectral interpretation, (2) the fitting-prediction engine for the refinement of the molecular parameters on the basis of the assigned transitions and the prediction of the rotational spectrum of the target molecule, (3) the GUI module that offers a powerful set of tools for a vis-à-vis comparison between experimental and simulated spectra, and (4) the new assignment tool for the assignment of experimental transitions in terms of quantum numbers upon comparison with the simulated ones. The implementation and the main features of VMS-ROT are presented, and the software is validated by means of selected test cases ranging from isolated molecules of different sizes to molecular complexes. VMS-ROT therefore offers an integrated environment for the analysis of the rotational spectra, with the innovative perspective of an intimate connection to quantum-chemical calculations that can be exploited at different levels of refinement, as an invaluable support and complement for experimental studies.

\section{INTRODUCTION}

Spectroscopic techniques provide a wealth of qualitative and quantitative information on the chemical and physical-chemical properties of molecular systems in a variety of environments. Among different spectroscopic methods, rotational spectroscopy, given its intrinsic high resolution and high sensitivity, is one of the most powerful tools for investigating the structure and dynamics of molecules and supramolecular systems in the gas phase. $^{1-10}$ As a matter of fact, rotational spectra contain a wealth of accurate information on structural, molecular, and spectroscopic parameters hardly or even not accessible from other experimental techniques. In particular, it is possible to retrieve rotational constants with an extremely high accuracy, which is then mirrored in the accuracy of the derived molecular geometries, to which they are directly related. ${ }^{2-5}$ However, the task of extracting these data, which starts with inverting the spectral observables, i.e., with the assignment of lines to an effective Hamiltonian, is challenging, time-consuming, and prone to errors.
The analysis of rotational spectra is strongly sped up by the use of suitable computer programs that assist in all phases of the procedure: from the assignment of the observed transitions to a set of rotational quantum numbers to their fitting within a given model Hamiltonian. To this purpose, a number of programs for the assignment of rotational spectra has been proposed over the years (see, e.g., ref 11), and the subject is still of great interest to the spectroscopic community. ${ }^{12-14}$ Remarkable examples of powerful and general-purpose rotational spectroscopy softwares are the AUTOFIT program, ${ }^{15}$ which allows an almost automatic assignment of broadband microwave (MW) spectra featuring complex molecular systems and/or conformational mixtures, ${ }^{16,17}$ together with PGOPHER ${ }^{12,18}$ and the widely used $\mathrm{AABS}^{19}$ and $\mathrm{JB}^{20}{ }^{20}$ packages for the analysis and fitting of rotational spectra. However, when challenging cases, e.g., showing high density of lines in the spectra, are to be dealt with, a significant step forward would be represented by the availability of computer programs

Received: May 23, 2017

Published: July 25, 2017 
that combine efficient assignment tools with accurate quantumchemical predictions of the spectroscopic parameters of interest. In fact, when large molecules or molecular complexes with different conformers and several low-lying vibrational states are to be investigated or when new chemical species that can be even unstable, like ions and radicals, are sought, then quantumchemical calculations provide an invaluable tool to guide experiments as well as to assist the spectral analysis. ${ }^{8,13-15,21-26}$ Nowadays, quantum-chemical calculations, ranging from density functional theory (DFT) or second-order Møller-Plesset perturbation theory (MP2) ${ }^{27}$ to the most refined composite schemes based on the coupled-cluster ansatz, also accounting for basis-set effects, ${ }^{28-34}$ play a fundamental role in modern approaches to rotational spectroscopy (see, e.g., refs 2, 21, and 35-39.).

In this work, we present the newborn rotational-spectroscopy module of the Virtual Multifrequency Spectrometer (VMS), VMS-ROT. Given the general philosophy of VMS and the leading role played by quantum-chemical calculations in modern rotational spectroscopy, unlike other software supporting the analysis of microwave (MW) spectra, VMS-ROT has been designed to be intimately related to theoretical calculations. Indeed, quantum-chemical predictions are incorporated as essential tools into the software and are used as starting points for guiding experiments and spectral interpretations. VMS is furthermore a software providing user-friendly access to the latest developments of computational spectroscopy ${ }^{40,41}$ and takes the Gaussian package ${ }^{42}$ as the reference source for spectroscopic data. As shown in the following, according to user's expertise in performing theoretical predictions, the program allows calculations of any kind: from standard geometry optimization and one-step frequency calculations up to highly accurate composite schemes also including anharmonicity effects.

In VMS, the computation of vibrational (infrared, Raman, vibrational circular dichroism) and electronic (one-photon absorption and emission and their chiral counterparts) spectra is already implemented and well tested. ${ }^{41}$ Electron spin resonance (EPR) spectroscopy has also been incorporated through the development of an effective interface between VMS and the E-SpiRes code. ${ }^{43}$ Very recently, VMS has been extended to deal with solid-state nuclear magnetic resonance (NMR) spectroscopy and simulate $1 \mathrm{D}$ and $2 \mathrm{D}$ NMR spectra. ${ }^{44}$ Furthermore, it has also been equipped with the Molecular Structure Refinement (MSR) code for the accurate determination of molecular structures by means of the semiexperimental approach. ${ }^{45,46}$ In addition to the computational module (VMSComp), which provides the support for the wide range of spectroscopies mentioned above, it also features VMS-Draw. ${ }^{4}$ This is a multiplatform graphical user interface (GUI), which offers to the user a powerful integrated environment for visualizing in real-time experimental and synthetic spectra, that performs their assignments and analyzes the results arising from the refinement of spectroscopic constants. VMS-Draw is therefore designed to support in a simple and intuitive way the analysis of computational simulations and the comparison of the results with experimental outcomes.

The sketch of the VMS-ROT program structure given in Figure 1 shows that it relies on four interconnected engines. The first one is the computational engine, which allows spectroscopic parameters to be computed from first principles in order to use them for the simulations of a synthetic spectrum from which the analysis of the experimental one can be started. It is worth

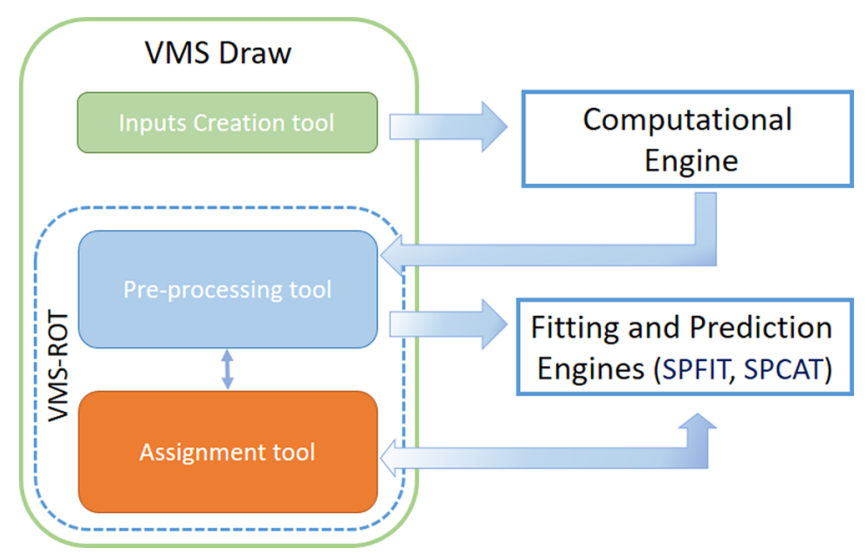

Figure 1. Block diagram showing the main components of VMS-ROT: (i) the computational engine allows the computation of the relevant spectroscopic parameters. (ii) Pickett's SPFIT and SPCAT programs are interfaced to the main program and work as fitting and prediction engines, respectively. (iii) The preprocessing tool of the VMS-ROT GUI provides an interface for the automatic creation of the input file for SPFIT/SPCAT starting from quantum-chemical calculations. (iv) The assignment tool allows loading simulated and experimental spectra for the assignment of the different transitions.

noticing that the high accuracy reached in the last years for computational strategies makes them invaluable aids for guiding experiments and assisting in their interpretation. The next tools within VMS-ROT are the fitting and prediction engines, which are in the form of Pickett's SPFIT/SPCAT programs. ${ }^{47}$ These have been chosen because they are widely used by the spectroscopic community (e.g., see refs $13,23-25$, and 48-53), and they can be considered a kind of standard, as demonstrated by the fact that spectroscopic databases, such as the Cologne Database for Molecular Spectroscopy (CDMS $)^{54}$ and the Jet Propulsion Laboratory (JPL) ${ }^{55}$ database, store data in the SPCAT format. The third module of VMS-ROT is a powerful and easy-to-use GUI, from which SPFIT and SPCAT input files can be intuitively prepared (e.g., with spectroscopic parameters computed from first-principles). Pickett's programs can also be run by a simple mouse-click, and their results are displayed and analyzed by a number of dedicated features. Finally, the GUI also gives access to the assignment tool that, as illustrated in the next section, can be used to assign a rotational spectrum recorded experimentally by comparing it with the simulated counterpart. The assignment tool incorporates all the manipulation features already present in VMS-Draw for assisting the spectral comparison, but it has been further equipped with a number of new additional and properly tailored tools. In practice, VMS-ROT aims at providing a one-pot solution for the analysis of rotational spectra, from the spectral assignment to the refinement of molecular parameters and spectral simulation, by working at the interface between experimental and computational rotational spectroscopy.

The paper is organized as follows: Section 2 provides a detailed description of the implementation of VMS-ROT and of its main features. Section 3 presents selected test cases, which aim at validating the program and demonstrating its user-friendliness and applicability to spectra recorded with different experimental setups and techniques. This also allows us to point out the flexibility of VMS-ROT for what concerns the level of quantumchemical calculations carried out to provide suitable guesses of the relevant spectroscopic data. Finally, conclusions and further developments are addressed in Section 4. 


\section{ROTATIONAL-SPECTROSCOPY MODULE OF VMS: IMPLEMENTATION AND TECHNICAL ASPECTS}

As briefly mentioned in the Introduction, VMS-ROT offers an integrated suite of tools for assisting the interpretation and understanding of rotational spectra: from the assignment of the spectral transitions and the refinement of spectroscopic constants to the prediction and simulation of the spectrum.

As is well known, the full assignment of a rotational spectrum requires finding the set of spectroscopic parameters of the most appropriate effective Hamiltonian able to completely describe the spectrum itself. From an operative point of view, this involves computing transition frequencies and intensities from a set of parameters, comparing them with their experimental counterparts to search for recognizable patterns, and making an initial assignment in terms of quantum numbers. Subsequently, the parameters are refined through a nonlinear least-squares fitting procedure to reproduce the assigned transitions. Next, the spectrum is recalculated, and the procedure proceeds iteratively until a close match between the experimental and the computed spectra is reached. In this assignment process, the selection of accurate initial guesses for the spectroscopic parameters, and above all, for the ground-state rotational constants, is one of the most critical steps in order to perform the first assignment and start the iterative analysis procedure. As anticipated in the Introduction, VMS-ROT is interfaced to quantum-chemical calculations for what concerns the determination of the initial guess of the spectroscopic parameters. For this purpose, the Gaussian package $^{42}$ is the reference source of the spectroscopic parameters, with the input for the SPFIT/SPCAT programs directly obtained from the quantum-chemical computations. However, a number of additional features, including a manual input, are present, and the extension to other quantum-chemical packages, like, e.g., CFOUR, ${ }^{56}$ is planned for the next releases of VMS-ROT. In particular, the use of quantum-chemical calculations is extremely flexible, with the following options available:

I. Geometry Optimization. Rotational constants corresponding to an optimized molecular structure are automatically extracted from Gaussian output and employed as starting guesses. It should be recalled that these constants actually refer to the equilibrium structure and not to the vibrational ground state, and hence, they may yield unreliable predictions. On the other hand, thanks to error compensation, certain computational models have been demonstrated to provide satisfactory estimates (e.g., see refs 57 and 58.).

ii. Anharmonic Calculations. At this stage, cubic and semidiagonal quartic force constants are computed, which are then used for calculating vibrational corrections to rotational constants (thus allowing the derivation of the vibrational groundand excited-state rotational constants) and centrifugal-distortion parameters. ${ }^{59,60}$ The computed quartic and sextic centrifugal distortion constants are taken as initial values for the vibrational ground state. An advanced feature of VMS-ROT allows rotational constants to be factored into the corresponding equilibrium values and vibrational corrections. At the occurrence (e.g., when using composite schemes, see below), they may be modified independently.

iii. Composite Schemes. Accurate predictions of equilibrium geometries, and therefore of the corresponding equilibrium rotational constants, can be effectively obtained by composite schemes. ${ }^{2,8,31,37,61}$ These schemes take the coupledcluster (CC) theory in the CC singles and doubles ${ }^{62}$ augmented by a perturbative treatment of triples $(\mathrm{CCSD}(\mathrm{T}))$ formulation $^{63}$ as a starting point and on top of it account for various missing contributions (e.g., finite basis set effects, core-valence correlation, ...), which are calculated at the highest possible level according to the size of the target system. Then, equilibrium rotational constants are corrected for vibrational contributions evaluated at a lower level of theory (e.g., DFT with hybrid or double-hybrid functionals). ${ }^{21,33,39}$ This approach has been profitably applied to a wide variety of molecules, ${ }^{21,35-37,64}$ and very recently, its efficacy has been demonstrated for molecular complexes as well. ${ }^{39,64}$ VMS-ROT offers the possibility to exploit such an approach to obtain very accurate estimates of rotational constants. In fact, it is possible to start from an anharmonic calculation carried out at the DFT level, which provides vibrational contributions and then load an equilibrium molecular structure (stored, for example, in a conventional xyz format) determined through a composite scheme. The rotational constants corresponding to this structure are automatically derived and vibrationally corrected according to the anharmonic calculation performed.

iv. Hyperfine Spectroscopic Parameters. When hyperfine interactions are present, the corresponding parameters, like nuclear quadrupole coupling constants and spin-rotation constants, are computed, automatically extracted from the Gaussian output file, and then employed as input data for the prediction of the hyperfine components.

For predicting transition intensities, dipole moments and rotational partition functions are required. The latter are calculated by the following equation: ${ }^{65}$

$$
Q_{r o t}=\frac{\pi^{1 / 2} T^{3 / 2}}{\sigma_{r}\left(\Theta_{a} \Theta_{b} \Theta_{c}\right)^{1 / 2}}
$$

where $\sigma_{r}$ is the rotational symmetry number, $T$ the absolute temperature, and $\Theta_{\alpha}$ the characteristic rotational temperature along the principal inertial axis $\alpha(=a, b, c)$, which is given by

$$
\Theta_{\alpha}=\frac{h^{2}}{8 \pi^{2} I_{\alpha} k_{\mathrm{B}}}
$$

with $I_{\alpha}$ denoting the principal moment of inertia about the corresponding axis. The characteristic rotational temperature is taken from the Gaussian output file; the user is asked to provide the temperature of interest, and then the rotational partition function is recalculated. In analogy to the spectroscopic parameters, the initial guesses for the components of the dipole moment along the principal inertia axes are taken from quantumchemical calculations. In this respect, it is worth noticing that when anharmonic calculations are performed, vibrationally averaged dipole moments are computed and used by VMSROT. Conversely, if the vibrationally averaged property is not available, VMS-ROT employs the equilibrium dipole moment components.

To assist the user in performing the quantum-chemical calculation of the relevant spectroscopic data, VMS-ROT is equipped with a build-in tool for automatically generating the required Gaussian input files starting from a molecular structure. Furthermore, in addition to the automatized approaches described above, there is also the possibility of removing or adding spectroscopic parameters, for example, the higher-order ro-vibrational interaction constants, as well as adjusting manually their values.

Concerning the fitting and prediction steps, VMS-ROT uses, respectively, the SPFIT and SPCAT programs developed by 
Pickett $^{47}$ and collected in the CALPGM suite of programs. Pickett's programs are very popular within the spectroscopic community, to the extent that they can be considered the de facto standard for rotational and ro-vibrational analysis (e.g., refs 13, 23-25, and 48-53.). However, it should be pointed out that sometimes the nonstraightforward encoding of the spectroscopic parameters may prevent their immediate use. For this reason, a graphical interface to the CALPGM suite of programs has been proposed some years ago with the aim of providing users with the symbols commonly employed for spectroscopic parameters. ${ }^{66}$ This idea has been further developed in VMS-ROT, thus providing an intuitive and user-friendly graphical user interface for SPFIT and SPCAT. This GUI allows creating the required input files for the two programs, running them, and analyzing their output at the end of the execution. In particular, once quantum-chemical calculations have been carried out, all the relevant spectroscopic parameters are loaded into dedicated forms of the GUI, and these data are then employed to generate the input files for SPFIT and SPCAT. The dialogue box for editing SPFIT and SPCAT inputs is shown in Figure 2, where

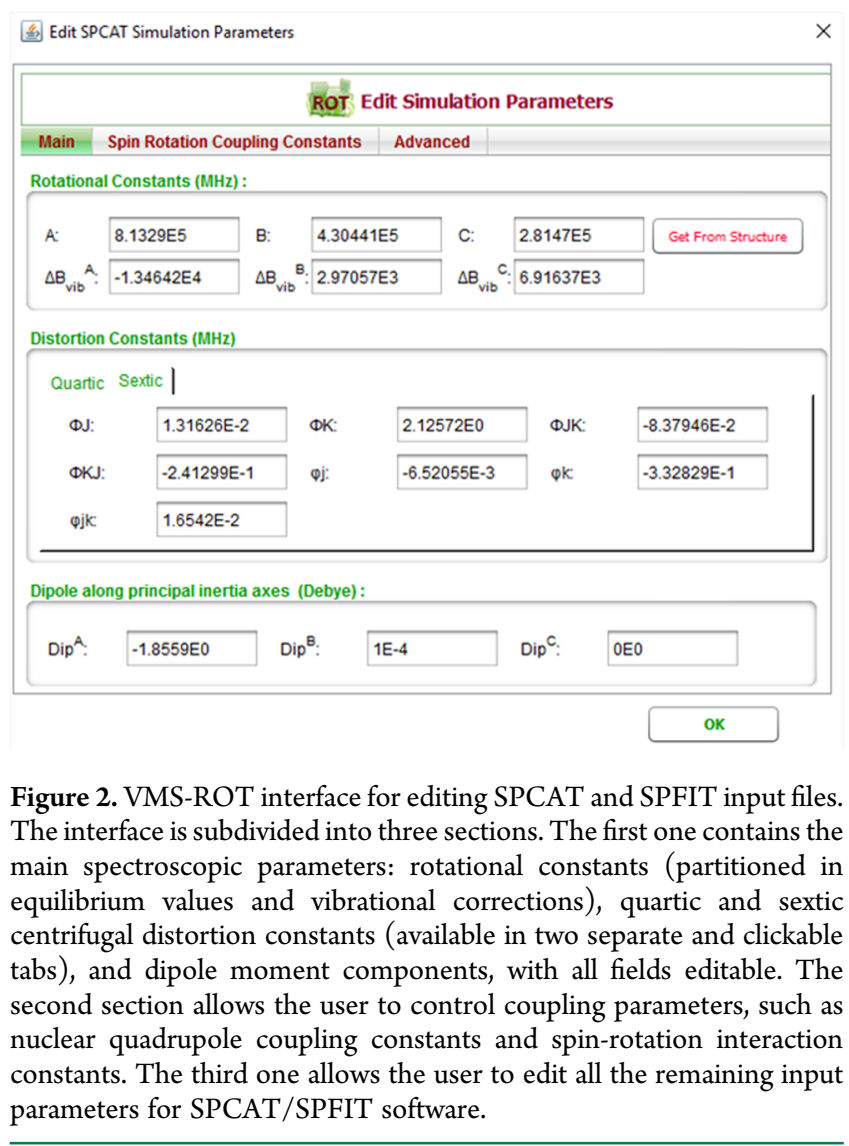

spectroscopic data have been directly loaded from a Gaussian output file and from which advanced features can be accessed, such as the separate treatment of equilibrium rotational constants and vibrational corrections or the possibility of getting the rotational constants corresponding to a molecular structure.

After all the required data have been provided, the SPFIT/ SPCAT program is executed, as a hidden process, by means of a simple mouse-click. The output detailing the progress of the computation is displayed into a dedicated output textbox, thus allowing the user to check the correct execution. At the end of the calculation, the output files are automatically loaded in VMS-
ROT: in the case of parameters refinement, the new values are displayed, whereas in the case of spectra prediction, the synthetic spectrum is shown in the plot area.

A number of tools are available for fast and efficient analyses of the fitting process: (i) At each iteration, the trend of the rootmean-square deviation of the fit is displayed in a dedicated plot. (ii) The correlation matrix among the spectroscopic parameters is graphically visualized by means of a gray scale plot, where color saturation provides an intuitive indication of the absolute value. The correlation matrix is certainly an important figure of merit in analyzing results, thus allowing one to quantify strong correlations between parameters that can lead to an illconditioned optimization problem. (iii) A panel is devoted to the analysis of the residuals between observed and calculated transition frequencies, thus allowing the user to pick up, at runtime, those that deviate more than a specified threshold. This feature is clearly useful in order to quickly detect wrongly assigned lines.

Once loaded in the plot area, the simulated spectrum can be edited by the user; for example, the stick spectrum can be convoluted with a suitable line shape function. Furthermore, in addition to the spectrum in absorbance and transmittance units, it is possible to obtain its first and second derivatives, the latter option being useful when it has to be compared with an experimental counterpart recorded by means of secondharmonic detection (i.e., by using the frequency modulation technique with the Lock-in amplifier tuned at twice the modulation frequency; this provides a good approximation of the second derivative of the natural line profile). The VMSComparison tool can be employed to compare spectra. This tool offers advanced manipulation utilities, which are useful for a fruitful spectral comparison, such as shifting and rescaling. Notably, if several species (e.g., conformers, tautomers, diasteroisomers, ...) can coexist under the experimental conditions, several computed spectra can be combined in a weighted mixture, according to the relative population of the different species.

Finally, VMS-ROT also offers an integrated environment for the assignment of an experimental spectrum based on the synthetic spectrum previously predicted. The appearance of the VMS-ROT Assignment tool is reproduced in Figure 3: The working area is subdivided into two horizontal panels, and the calculated spectrum is displayed in the top one, whereas the experimental trace can be loaded in the lower panel. Clearly, VMS spectral manipulation tools are available also for editing the experimental spectrum, a feature that may be useful, for example, for calibration purposes. Furthermore, a peak-picking function is also available at the occurrence in order to locate local maxima above a given threshold set by the user. When both spectra are loaded into the corresponding panels, the assignment feature can be accessed by clicking on the proper item of the toolbar, which leads to the opening of the register of the assigned transitions. For each assigned transition, this table contains the list of the upper and lower level quantum numbers, the measured and calculated frequency together their difference, the estimated experimental uncertainty, and the weight of a transition within a blend of overlapped lines. The assignment of a given experimental line, based on the comparison with the theoretical spectrum, can be performed in a manual or an automatic mode. The former option allows the user to insert manually all the details of the transitions to be assigned. Conversely, in the automatic mode, the assignment can be easily performed by first selecting a peak in the theoretical spectrum and then by clicking 


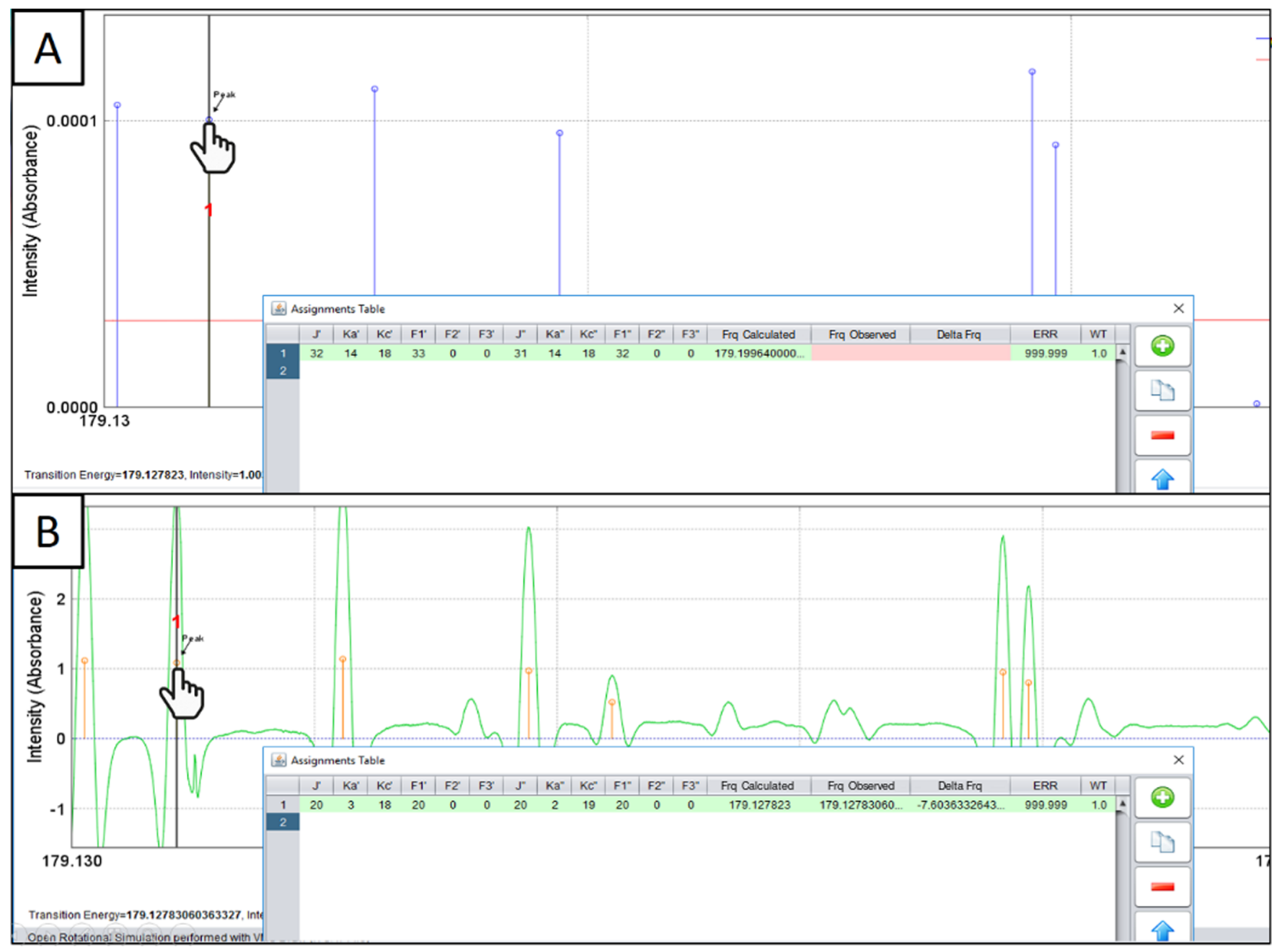

Figure 3. Assignment tool of VMS-ROT. (A) Selection of a transition in the calculated spectrum adds a new line in the assignment table, with quantum numbers and calculated frequency of the selected peak. (B) The assignment is completed by selecting a transition of the experimental spectrum, which is added to the assignment table and the relevant information is also updated. Graphical annotations in both spectra (calculated and experimental) highlight the peaks involved during the assignment or selected in the assignment table.

on the experimental line that the user wishes to assign, as exemplified in Figure 3. It is worth noticing that when a peak is selected, all the relevant information is detailed in the status bar. This includes the frequency and the intensity of the transitions and, for the calculated spectrum, the set of quantum numbers of the transition.

Once a set of transitions has been assigned, it is possible to perform the spectral fitting by clicking on the <Fitting transition $>$ button available in the toolbar of the assignment window. As anticipated, in VMS-ROT the fitting process is performed by an embedded version of the SPFIT program, which requires two input files, namely, a parameter file defining the spectroscopic parameters to be refined (the .PAR file) and a line-list file collecting the assigned transitions (the .LIN file). These input files are automatically generated by VMS-ROT starting from the last set of parameters employed to perform the simulation of the spectrum and from the list of assigned transitions, respectively. Before performing the fit, a dedicated textbox is available, where the user can modify the rotational parameters file, for example, by setting the variability of the spectroscopic constants during the fitting (although sensible values are used by default). A peculiar feature of VMS-ROT is that when the fitting process is completed SPCAT is run as a background process with the newly determined parameters, the corresponding calculated spectrum is updated, and so are the data stored in the record of assigned transitions. By clicking on the $<$ Simulation $>$ button, the updated spectrum is visualized in the top horizontal panel. As already described, a number of tools are available to check the outcomes of the fit. At this point, the spectral assignment can proceed further and the process iterated until the assignment of the experimental spectrum is considered satisfactory.

\section{RESULTS AND DISCUSSION}

Validation of VMS-ROT and illustration of its efficiency and versatility have been performed by means of a number of case studies: pyridine, methyl- $\beta$-D-xyloside, fenchone, and acetonitrile have been selected for isolated molecules, while pyridine $\cdots$ ammonia (PYR $\cdots \mathrm{NH}_{3}$ ) and difluoromethane $\cdots$ tert-butyl-alcohol (DFM…TBA) have been considered for clusters. In the following, the initial assignment of the MW spectra, one of the most challenging steps of the rotational spectra analysis, has been performed by comparing the experimental spectrum with that simulated by using spectroscopic and molecular parameters from quantum-chemical calculations. In most cases, the computations required to generate the first guesses of rotational constants, 
hyperfine parameters, and dipole moment components have been performed by the Gaussian software. ${ }^{42}$

The rotational spectra of the considered molecules have been taken from different sources, thus showing the flexibility of VMSROT in different scenarios. For example, in the case of pyridine, the pure rotational spectrum in the range $0-20.4 \mathrm{GHz}$ has been synthesized starting from the spectroscopic parameters determined by Heineking et al. ${ }^{67}$ In particular, the SPCAT input files (namely, the .VAR and .INT files) have been generated manually by using the dedicated tool of VMS-ROT. Then, SPCAT has been run by a simple mouse-click on a toolbar button. The output file (.CAT) obtained at this stage has been loaded in the Experimental panel of the VMS-ROT Assignment tool and convoluted with a Gaussian line-shape function having a halfwidth at half-maximum (HWHM) of $50 \mathrm{kHz}$. In this way, it has been possible to obtain a synthetic spectrum that closely matches its experimental counterpart. This example shows two nice features of VMS-ROT: (i) The program is able to read and automatically load SPCAT output files (even by a simple drag and drop of the file), thus maintaining full file format compatibility and providing access to existing analyses. (ii) It is possible to generate experimental-like spectra starting from accurately determined spectroscopic constants. In the subsequent step, a reanalysis of the pyridine spectrum so generated has been performed. For this purpose, the initial guesses of the ground-state rotational constants have been obtained from the so-called "cheap" composite scheme for equilibrium geometry. ${ }^{2,68}$ By means of this approach, it is possible to obtain accurate equilibrium geometries, with an accuracy of $\pm 0.001 \AA$ and $\pm 0.2^{\circ}$ for bond lengths and angles, respectively, which is mirrored in the corresponding equilibrium rotational constants. By using the dedicated functionality of VMS-ROT, equilibrium rotational constants have been extracted from the highly accurate geometry and then automatically corrected for vibrational effects computed by using the B2PLYP double hybrid functional ${ }^{69}$ in conjunction with the m-aug-cc-pVTZ- $d \mathrm{H}$ basis $\operatorname{set}^{70}(-d \mathrm{H}$ indicates that polarization functions have been removed for the hydrogen atom) and augmented by Grimme's DFT-D3 dispersion corrections. ${ }^{71,72}$ The computational results, taken from ref 38 , are reported in Table 1 together with their experimental counterparts.

Table 1. Rotational Constants and ${ }^{14} \mathrm{~N}$ Nuclear Quadrupole Coupling Constants of Pyridine

$\begin{array}{lcc} & \text { Theory }^{a} & \text { Experiment }^{b} \\ A / \mathrm{MHz} & 6032.5 & 6039.2516(6) \\ B / \mathrm{MHz} & 5809.6 & 5804.9116(6) \\ \mathrm{C} / \mathrm{MHz} & 2958.8 & 2959.2117(6) \\ \chi_{\mathrm{aa}} / \mathrm{MHz} & -4.99 & -4.908(3) \\ \chi_{\mathrm{bb}} / \mathrm{MHz} & 1.47 & 1.434(3) \\ \chi_{\mathrm{cc}} / \mathrm{MHz} & 3.52 & 3.474(3)\end{array}$

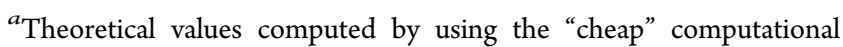
scheme augmented by vibrational corrections at the B2PLYP-D3/maug-cc-pVTZ- $d \mathrm{H}$ level, see ref $39 .{ }^{b}$ From ref 67 . Values in parentheses correspond to one standard deviation.

The theoretical spectrum has been generated by using the computed spectroscopic parameters of Table 1. A detail, i.e., the hyperfine structure of the $J=1_{0,1}-0_{0,0}$ rotational transition, is presented in Figure 4, where the quantum number $F$ comes from the $J+I$ coupling scheme, with $I$ being the ${ }^{14} \mathrm{~N}$ nuclear spin $(I=$ $1)$. The comparison of experimental and theoretical spectra shows a good agreement, which has made it possible to easily start the assignment of the experimental features. This agreement also demonstrates the accuracy of the computational approach adopted and how it can be straightforwardly exploited by using VMS-ROT, hence speeding up the rotational analysis process. For pyridine, 30 transitions have been assigned and then used by the SPFIT program integrated in VMS-ROT for the fitting procedure, finally leading to the same parameters as those reported by Heineking et al. ${ }^{67}$

As a second test, we have selected the bicyclic terpenoid fenchone molecule $\left(\mathrm{C}_{10} \mathrm{H}_{16} \mathrm{O}\right)$, which has been investigated by Loru et al. by using chirped-pulse Fourier transform microwave (CP-FTMW) spectroscopy in the $2-8 \mathrm{GHz}$ frequency region. ${ }^{73}$ The initial values for rotational constants and dipole moment components have been obtained for the equilibrium configuration of the molecule at the MP2 level of theory ${ }^{27}$ in conjunction with the Pople's style triple- $\zeta 6-311++\mathrm{G}(d, p)$ basis set. $^{74}$ On the basis of the spectrum simulated by using the spectroscopic parameters at the aforementioned level, this assignment has started with the identification of some low $J b$ type transitions. These have been fitted using Watson's $A$ reduction Hamiltonian in the $I^{r}$ representation ${ }^{75}$ to yield an improved set of rotational constants, which has been used to compute a new simulated spectrum in closer agreement with the experimental one. Hence, further assignments have been performed and the fitting procedure carried out, and this time also refining the quartic centrifugal distortion constants, whose estimates have been obtained at the MP2/6-311++G $(d, p)$ level as well. The assignment-fitting-prediction steps have been iterated up to the assignment of all the reported 150 measured transitions. It is important here to recall that, once having performed the fitting, VMS-ROT automatically calls, as a background process, SPCAT for the prediction of the new simulated spectrum that is then displayed in the corresponding panel of the GUI, and the list of assigned transitions (in particular, the computed transition frequencies and the observed-calculated differences) is uploaded as well. Hence, the user can stay concentrated on the analysis of the spectrum rather than on a manual in-and-out among different programs. An example of the VMS-ROT GUI showing the comparison between the synthetic and experimental rotational spectrum of fenchone in the $4.24-4.28 \mathrm{GHz}$ frequency range is given in Figure 5. The experimental transition frequencies have been retrieved from the CP-FTMW spectrum by using the peakpicking tool, integrated in VMS-ROT. It should be noted that, while rotational and centrifugal distortion constants have been refined, intensities have been predicted by using the computed dipole moment components. This test also illustrates how VMSROT is able to extract the relevant spectroscopic parameters, obtained from a geometry optimization, from a Gaussian output file, hence exploiting quantum chemical-calculations at very different levels of refinement.

The rotational spectrum of methyl- $\beta$-D-xyloside has been considered as a further test case in order to show the flexibility and ease of use of VMS-ROT. This molecule was first investigated by Alonso and co-workers by combining CPFTMW spectroscopy with picosecond-laser ablation. ${ }^{76}$ The analysis of the broadband rotational spectrum in the $6-12 \mathrm{GHz}$ range led to the identification of the exoanomeric effect and hydrogen bonding as the main factors controlling the conformational behavior of the archetypal methyl- $\beta$-D-xyloside molecule. In this case, the relatively large size of the molecule allowed the assignment only of very low- $J$ rotational transitions, hence 


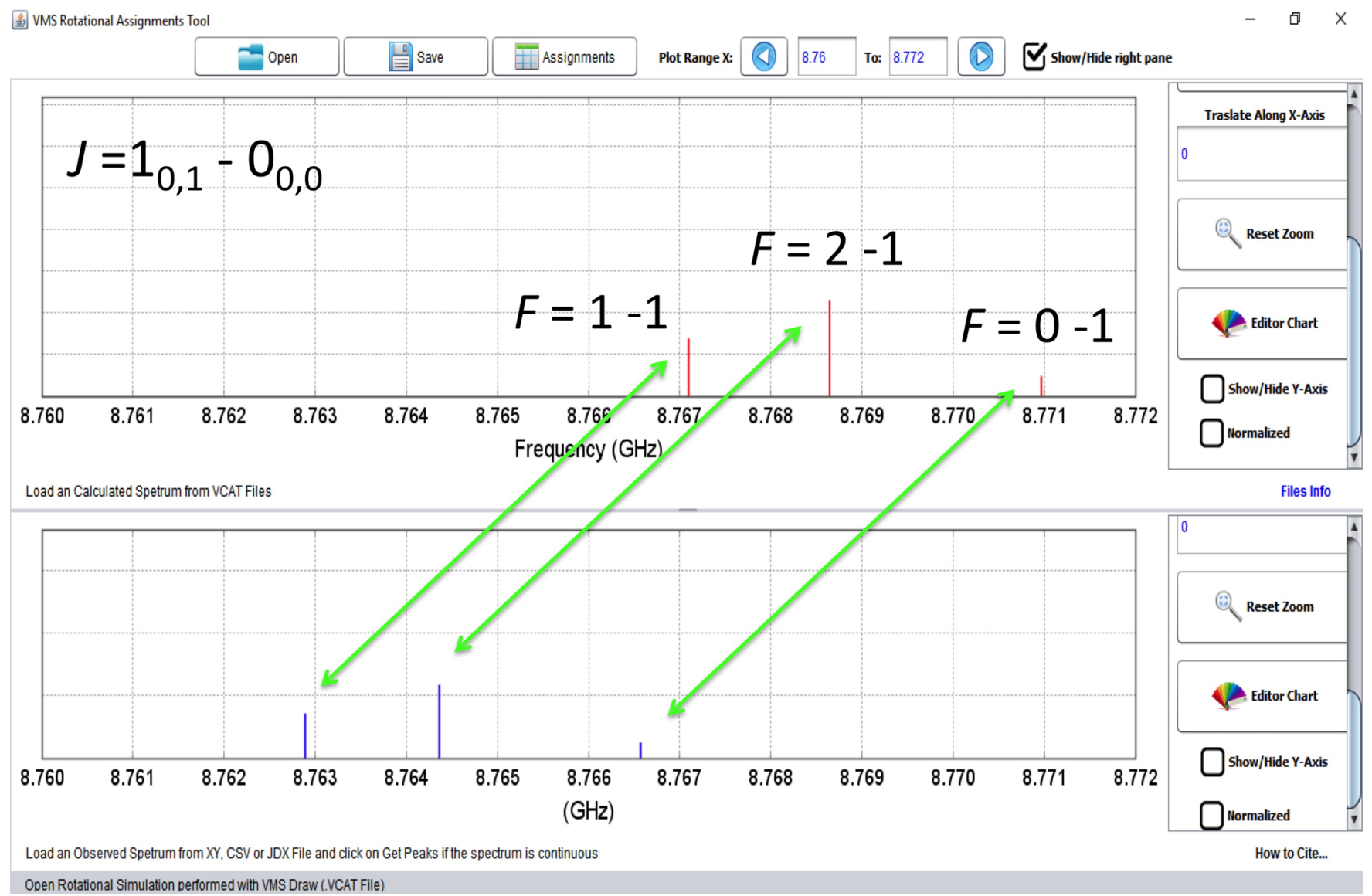

Figure 4. Comparison of the experimental and simulated (see text) spectra of pyridine in the $8.760-8.772 \mathrm{GHz}$ range: hyperfine structure of the $J=1_{0,1}$ $-0_{0,0}$ transition. The theoretical spectrum (top panel) has been synthesized by correcting the equilibrium rotational and nitrogen-quadrupole coupling constants evaluated using the "cheap" composite scheme with vibrational corrections at the B2PYLP-D3/m-aug-cc-pVTZ- $d \mathrm{H}$ level. The experimental spectrum is shown in the bottom panel.

limiting the determination of the spectroscopic parameters to the ground-state rotational constants. For this molecule, the geometry optimization of the most stable conformer has been here carried out at the MP2/6-311++G $(d, p)$ level to obtain the corresponding equilibrium rotational constants. Because of error cancellation effects, the predicted rotational constants turned out to be in good agreement with the ground-state rotational constants. From the preliminary simulation, it has been possible to assign all the 64 reported experimental transitions. Their use in the fitting led to a refinement of the computed parameters and to a simulated spectrum that closely matches the measured one, as illustrated in Figure 6a. This figure shows the comparison between the experimental and simulated spectra over the whole 6-12 GHz spectral region. We note that, even if not displayed, the transitions of the experimental and theoretical spectra can be labeled. These labels identify the line in the record of assigned transitions from which the assignment can be removed or edited manually. A magnified view of the methyl- $\beta$-D-xyloside spectrum in the $8.80-8.86 \mathrm{GHz}$ range is presented in Figure $6 \mathrm{~b}$, where the synthetic trace has been convoluted with a Gaussian function with a HWHM of $100 \mathrm{kHz}$. As shown, the agreement is excellent for both line positions and intensities. Clearly, the ground-state rotational constants obtained from the fitting process are the same as those determined in ref 76 .

The above test cases show that VMS-ROT is able to assist all the steps in the analysis of the rotational spectra of asymmetric rotor molecules. Yet, the program is not limited to this class of rotors, also providing support for symmetric and linear molecules. To illustrate this feature, the rotational spectrum of acetonitrile $\left(\mathrm{CH}_{3} \mathrm{CN}\right)$ is taken as an example. In this case, the pure rotational spectrum in the range $160-190 \mathrm{GHz}$ has been synthesized starting from the data collected in the CDMS. ${ }^{77-79}$ The .CAT file, downloaded from the CDMS Web site, has been directly loaded into the experimental panel of the VMS-ROT Assignment tool. The resulting stick-spectrum has then been convoluted with a Voigt line-shape function having a HWHM of $500 \mathrm{kHz}$, thus obtaining, as in the case of pyridine, a synthetic spectrum that (almost) exactly matches its experimental counterpart. This shows another powerful feature of VMSROT, namely, that it is possible to load spectra by interfacing it with different databases dedicated to spectroscopy, such as CDMS,${ }^{54}$ HITRAN, ${ }^{80}$ GEISA, ${ }^{81}$ and JPL. ${ }^{55}$ To analyze the $\mathrm{CH}_{3} \mathrm{CN}$ spectrum, vibrational corrections to rotational constants as well as quartic and sextic centrifugal distortion constants have been computed at the B2PLYP/m-aug-cc-pVTZ- $d \mathrm{H}$ level. These have been used in conjunction with equilibrium rotational constants derived from ref 82 to obtain the synthetic spectrum reported in Figure 7 . The theoretical spectrum results to be shifted by a few $\mathrm{GHz}$ (less than $2 \%$ in relative terms) from the experimental trace, but the overall trend is very well reproduced, thus allowing an unambiguous assignment. In fact, by performing a fit solely based on four assigned transitions (two for each of the two sub-bands), the refined spectroscopic constants $\left(B, D_{J}, D_{J K}\right)$ have led to a simulated spectrum in close agreement with the 


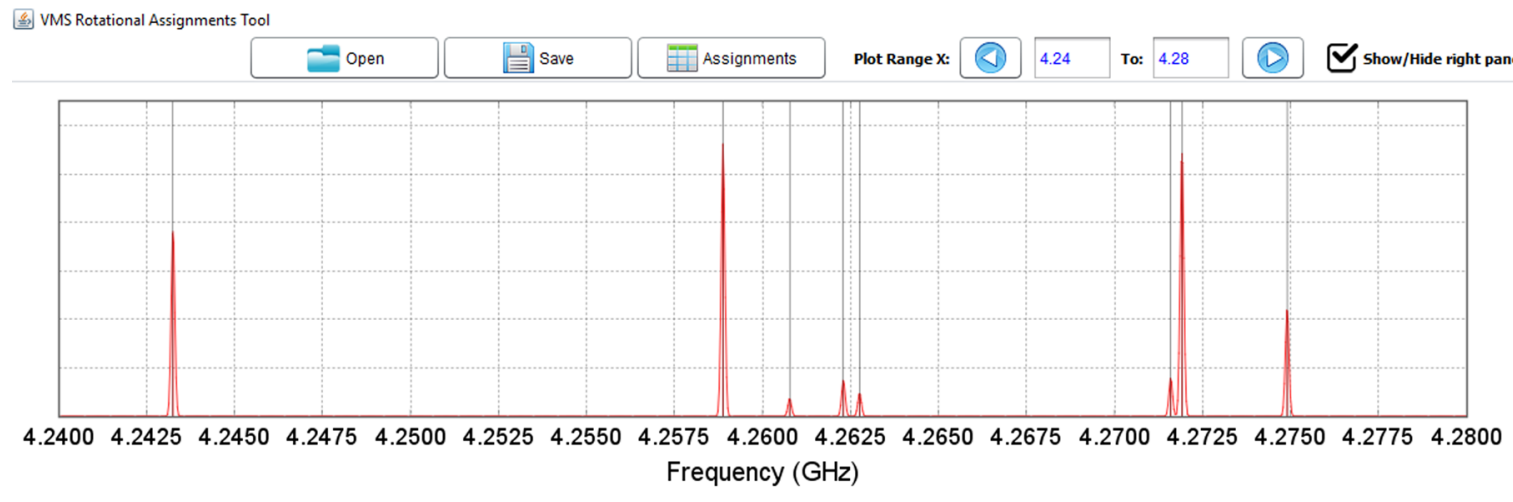

Load an Calculated Spetrum from VCAT Files
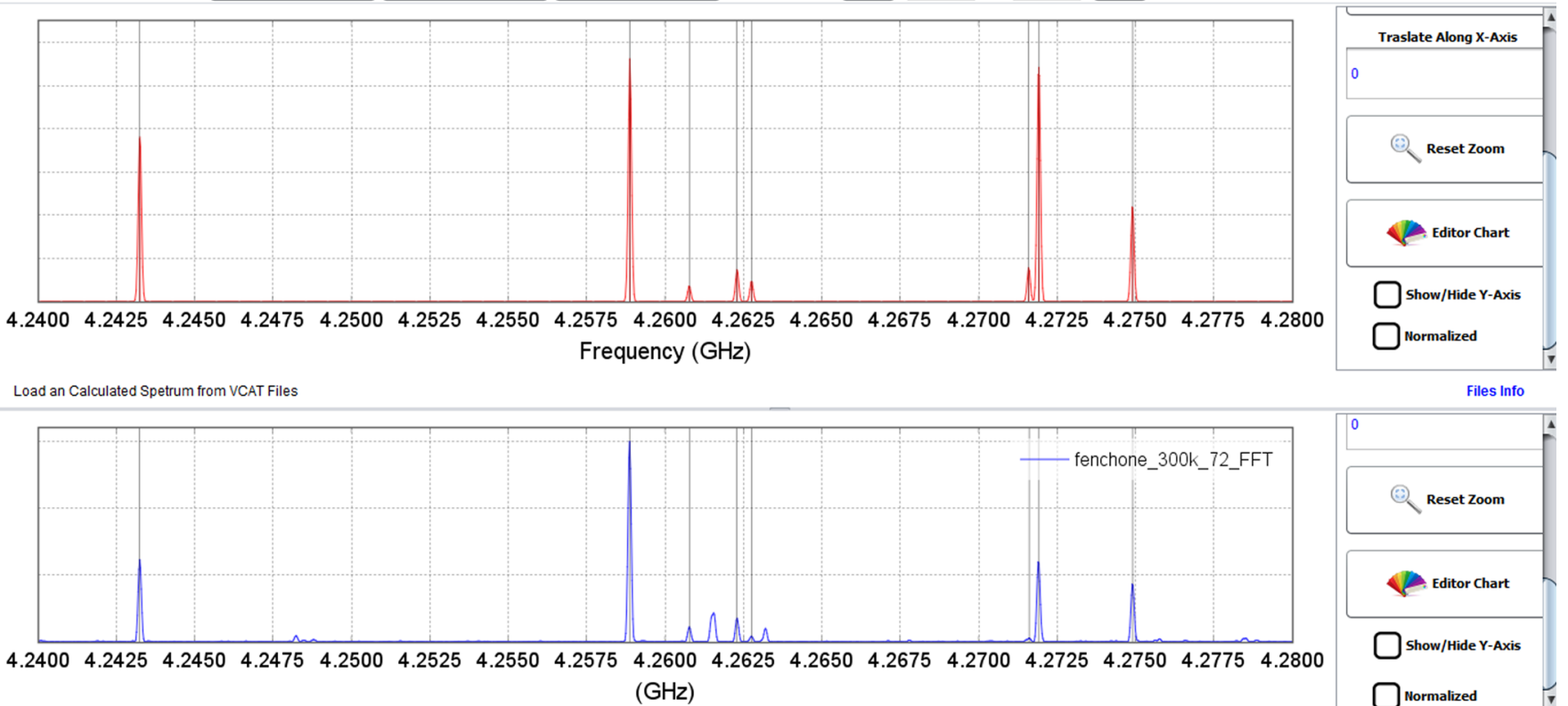

Load an Observed Spetrum from XY, CSV or JDX File and click on Get Peaks if the spectrum is continuous

Files Info

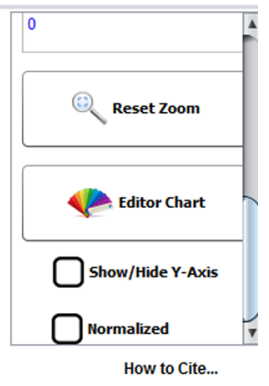

Open Rotational Simulation performed with VMS Draw (.VCAT File)

Figure 5. GUI interface of VMS-ROT showing the comparison between the synthetic (top panel) and CP-FTMW (bottom panel) rotational spectra of fenchone after the refinement of the spectroscopic parameters. Solid gray vertical lines denote the assigned transitions. Intensities are simulated by using the computed dipole moment components.

experimental one, as shown in Figure 8. Starting from this first guess, it has been possible to assign 19 lines with rotational quantum numbers in the $9 \leq J \leq 10$ and $0 \leq K \leq 9$ ranges. At this point, a comment on the analysis of symmetric and linear rotor spectra is deserved. In fact, during the implementation of VMSROT, we realized that the commercial versions of the Gaussian software do not correctly generate the .PAR (or, equivalently, .VAR) file required to run Pickett's software. In particular, the keyword "output = Pickett" produces a wrong third line of the input file, and spectroscopic parameters are always written with reference to asymmetric rotors by using Watson's Hamiltonian in the $A$ reduction. While these issues will be solved in the forthcoming distributions of the Gaussian software, they do not represent a real problem for VMS-ROT. In fact, VMS-ROT automatically recognizes the kind of rotor it is dealing with, and in the case of symmetric or linear molecules, it adjusts the parameter encoding, while a message prompts the user to manually modify the wrong entry in the third line read from the Gaussian output file in order to recover the right intensity alternation.

As anticipated, VMS-ROT has been validated also for the analysis of rotational spectra of molecular complexes, focusing on the PYR $\cdots \mathrm{NH}_{3}$ and DFM $\cdots$ TBA dimers, which have been recently investigated by Spada et al. ${ }^{39,64}$ by means of an integrated experimental-theoretical approach joining Fourier Transform Microwave spectroscopy (FTMW) to state-of-the-art quantum-chemical calculations. Concerning the DFM $\cdots$ TBA complex, the initial assignment has been carried out on the basis of the spectrum simulated by using the spectroscopic parameters computed at the B2PLYP-D3/m-aug-cc-pVTZ- $d \mathrm{H}$ level. In the case of PYR $\cdots \mathrm{NH}_{3}$, initial guesses for the rotational constants have been derived correcting the equilibrium rotational constants corresponding to the geometry evaluated by means of the "cheap" geometry scheme with vibrational corrections at the
B2PLYP-D3/m-aug-cc-pVTZ- $d$ H level. The latter level of theory has been employed for computing quartic centrifugal distortion constants, whereas the "cheap" scheme has been used for nuclear quadrupole coupling constants as well. In order to overcome the problems originated by the splitting of the rotational transitions due to Doppler effect (arising from the coaxial arrangement of the molecular beam expansion and the resonator axis), each peak of the spectrum loaded in the experimental spectrum panel of VMS-ROT actually corresponds to the average of the two components in which each line is split. In this respect, a tool allowing the real time derivation of the Doppler unshifted peak position is being developed in order to handle this kind of spectra without any additional external user manipulation. The assignment of the spectra of DFM $\cdots$ TBA and PYR $\cdots \mathrm{NH}_{3}$ has followed the procedure described in refs 39 and 64, respectively, clearly obtaining the same spectroscopic parameters. The spectrum of the DFM $\cdots$ TBA complex in the $6.5-18.5 \mathrm{GHz}$ region, obtained after parameter refinement, is presented in Figure 9, whereas that of PYR $\cdots \mathrm{NH}_{3}$, showing the nine nitrogen-quadrupole coupling components within the two internal rotation $E$ and $A$ levels, between 14.7350 and $14.7375 \mathrm{GHz}$, is shown in Figure 10. In both cases, the simulated spectrum reproduces well the experimental one in terms of both line positions and intensities. The latter ones have been predicted by using vibrationally averaged dipole moments computed at the B2PLYP-D3/m-augcc-pVTZ- $d \mathrm{H}$ level of theory. As shown, VMS-ROT is well suited also for analyzing the rotational spectra of molecular adducts, in addition to those of isolated molecules.

\section{CONCLUSIONS AND OUTLOOK}

VMS-Draw is a recently developed software for computational spectroscopy, which allows computing a wide variety of spectroscopic properties and visualizing the corresponding spectra. This powerful graphical interface is purposely tailored 


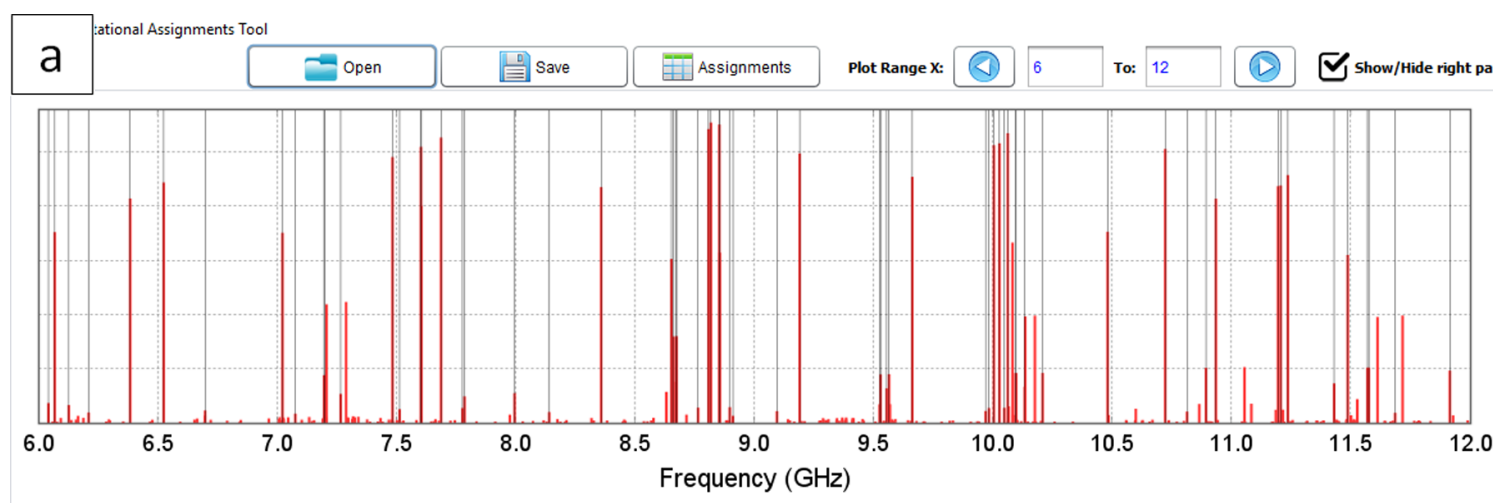

$-\square \times$

Load an Calculated Spetrum from VCAT Files

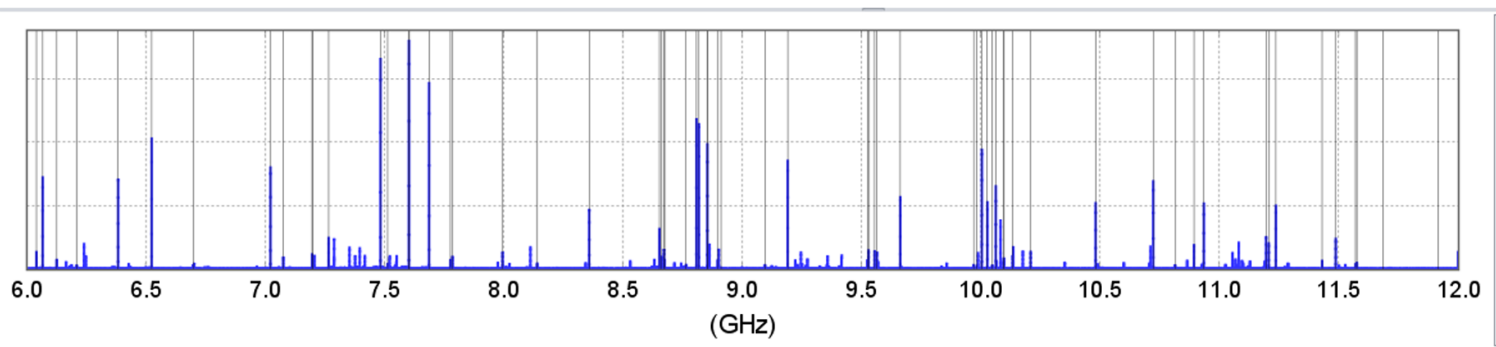

Load an Observed Spetrum from XY, CSV or JDX File and click on Get Peaks if the spectrum is continuous

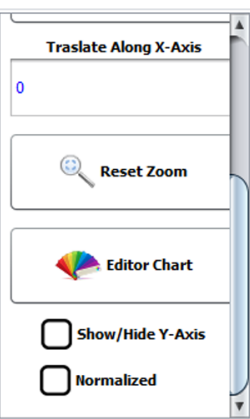

Files Info

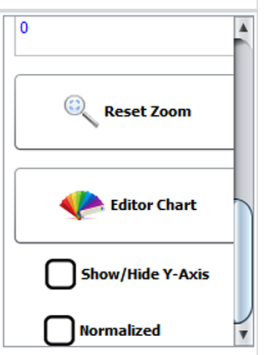

How to Cite...

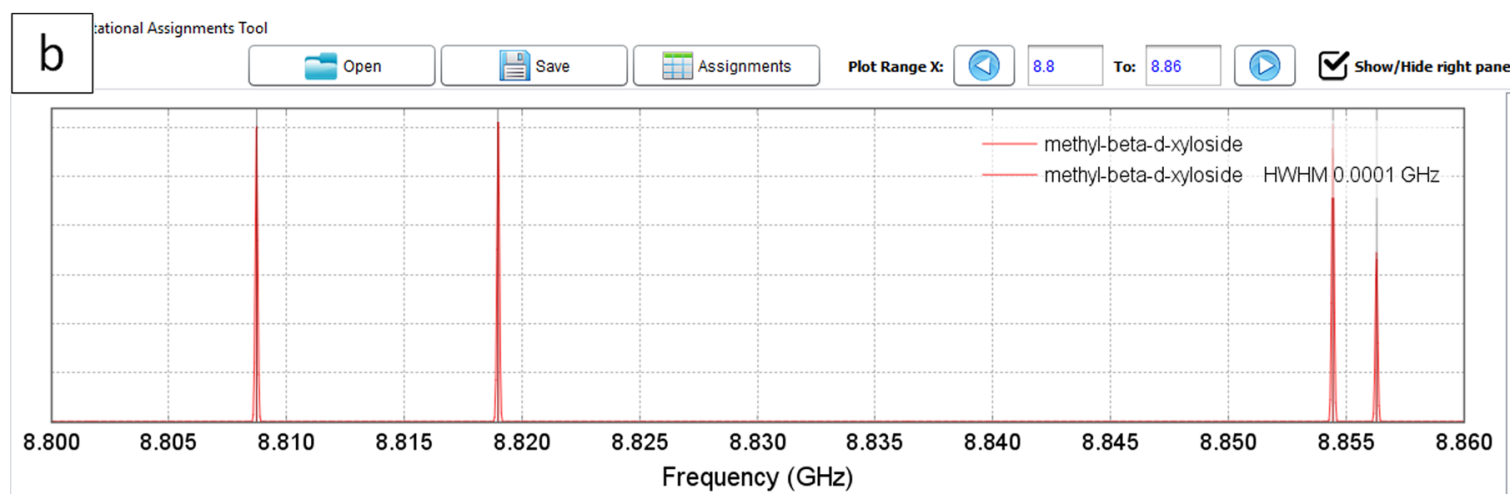

Load an Calculated Spetrum from VCAT Files
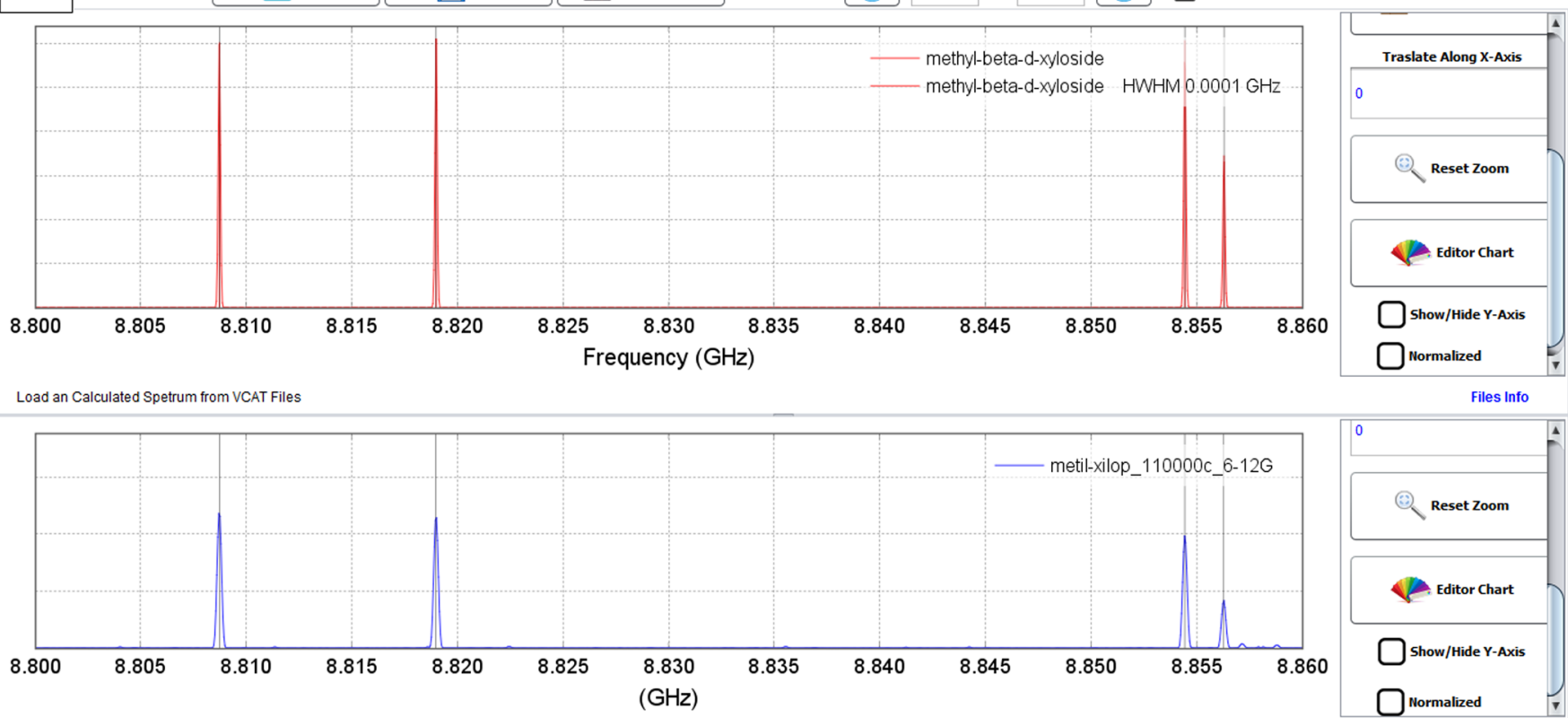

How to Cite.

Open Rotational Simulation performed with VMS Draw (.VCAT File)

Figure 6. (a) Synthetic (top panel) and CP-FTMW experimental (bottom panel) spectrum of methyl- $\beta$-D-xyloside between 6 and 12 GHz analyzed employing VMS-ROT. (b) A magnified view of the $8.80-8.86 \mathrm{GHz}$ spectral region. The computed spectrum (upper panel) has been convoluted with a Gaussian function having a HWHM of $100 \mathrm{kHz}$.

for automatically extracting the information from the output of a quantum-chemical calculation, with the Gaussian software taken as the standard source, building the simulated spectrum, and visualizing all relevant information in an intuitive format. In addition, it presents a number of advanced tools for spectra manipulation and comparison, thus assisting and speeding up their interpretation. Therefore, VMS-Draw has been designed for both experienced users (permitting a straight and deeper interpretation of the computed quantities in the form of $2 \mathrm{D}$ and $3 \mathrm{D}$ plots or spectra) and nonspecialists (permitting an intuitive visualization and comparison with the experimental traces). Up to now, the program has been devised to treat several 

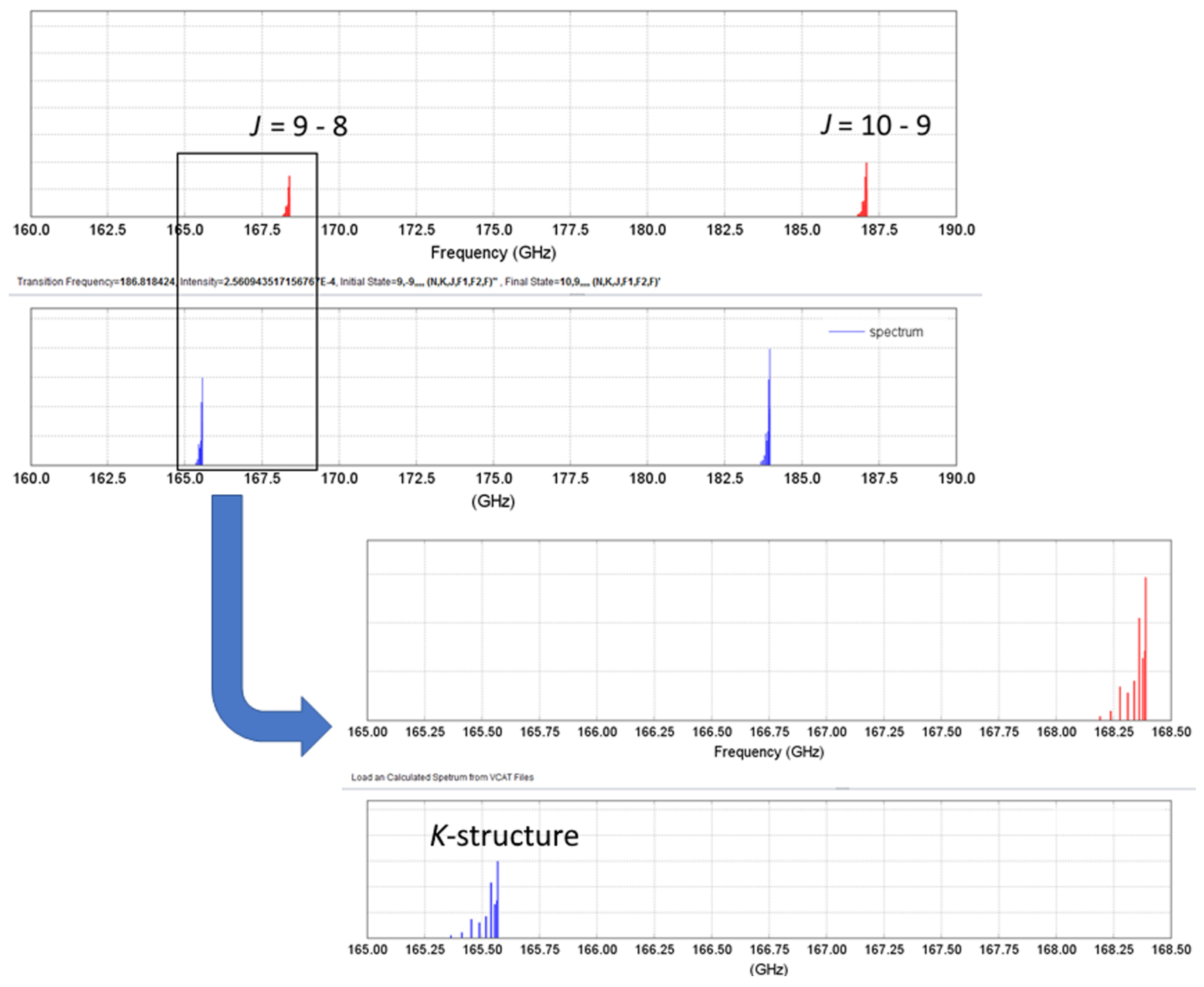

Figure 7. Pure rotational spectrum of $\mathrm{CH}_{3} \mathrm{CN}$ in the $160-190 \mathrm{GHz}$ frequency range: the $J=9-8$ and $J=10-9$ rotational transitions. The theoretical spectrum (in red, top panel) has been synthesized by using the equilibrium rotational constants from ref 82 corrected for vibrational contributions at the $\mathrm{B} 2 \mathrm{PLYP} / \mathrm{m}$-aug-cc-pVTZ- $d \mathrm{H}$ level. A magnified view of the 165-168.50 interval shows the close correspondence between the quantum chemical predictions and the experimental features (in blue, bottom panel).

types of vibrational and electronic spectra, as well as EPR and, more recently, NMR spectra.

With the aim of extending further the spectral range covered by the virtual multifrequency spectrometer, in this work, we have presented the newborn module, VMS-ROT, specifically designed for rotational spectroscopy. In addition to the possibility of simulating the high-resolution rotational spectra of molecules and molecular complexes, it provides powerful tools for carrying out the assignment of an experimental spectrum and the refinement of the spectroscopic parameters, such as rotational constants, centrifugal distortion parameters, and hyperfine coupling terms. For the fitting and prediction steps, VMS-ROT is interfaced with Pickett's SPFIT and SPCAT programs that, over the years, have become the de facto standard for rotational- and ro-vibrational spectroscopy. VMS-ROT offers a dedicated interface to these two programs, thus avoiding the cumbersome encoding of spectroscopic parameters. Furthermore, SPFIT and SPCAT can be directly run from the VMSROT GUI as background processes, and their output is automatically visualized, in terms of both the obtained spectroscopic parameters and the corresponding synthetic spectrum. The program also offers a complete set of tools for the spectral assignment on the basis of the comparison between computed and experimental spectra. By pursuing the general VMS-Draw philosophy and following the recent trend in the field of rotational spectroscopy, VMS-ROT has been designed to work at the interface between computational and laboratory spectroscopic techniques. Quantum-chemical calculations can be exploited at various levels of theory to obtain first estimates of the relevant spectroscopic parameters. In fact, the program can handle different working scenarios for quantum-chemical calculations, from simple geometry optimizations to refined composite schemes, also accounting for anharmonic effects. Then, it automatically extracts the spectroscopic parameters and performs the spectral simulation.

VMS-ROT has been validated against different test cases, including both isolated molecules, like pyridine, fenchone, methyl- $\beta$-D-xyloside, and acetonitrile, as well as molecular complexes, in particular, DFM $\cdots$ TBA and PYR $\cdots \mathrm{NH}_{3}$. These tests have demonstrated the flexibility and robustness of the 


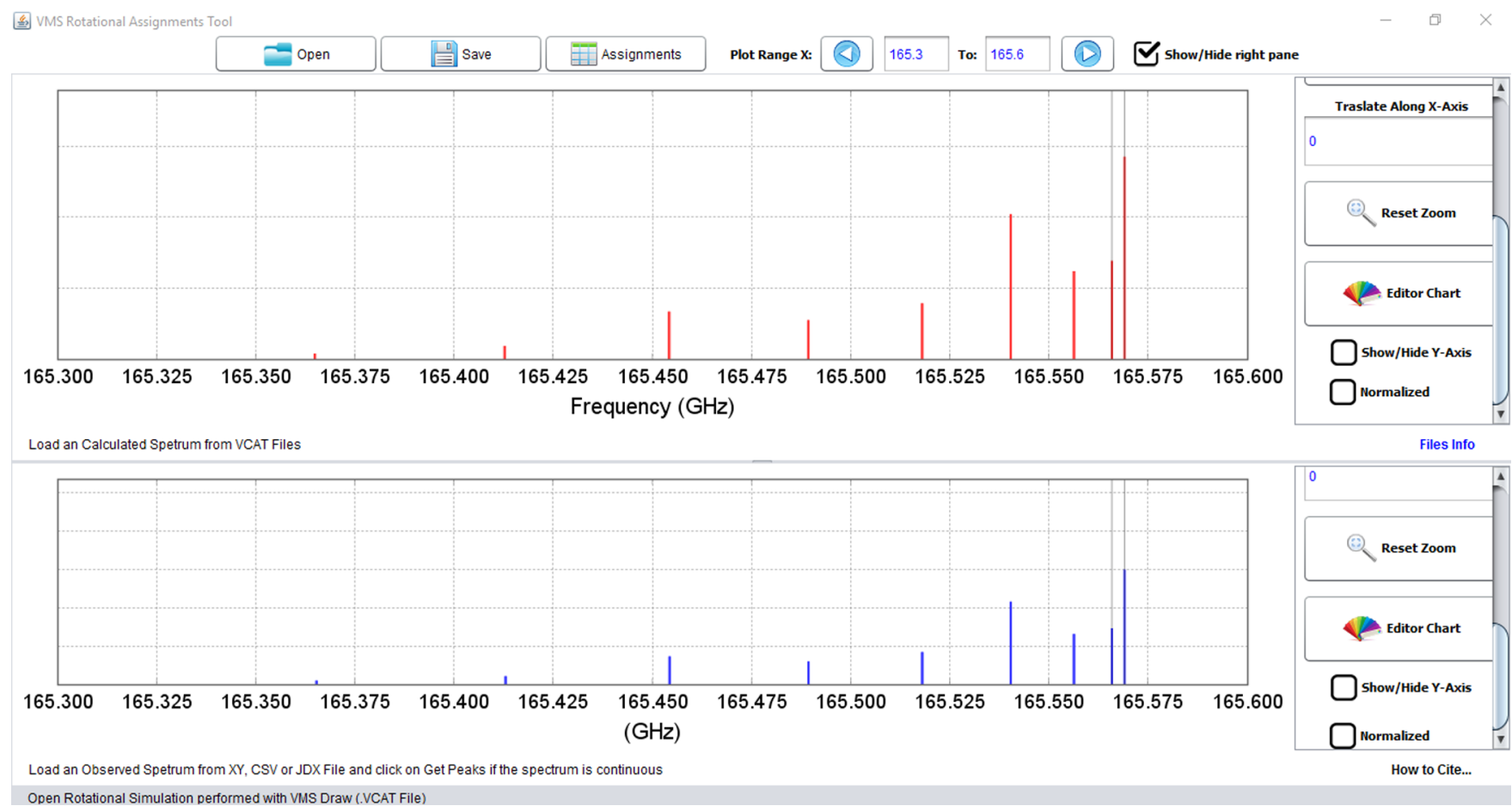

Figure 8. Pure rotational spectrum of $\mathrm{CH}_{3} \mathrm{CN}$ in the $165.3-165.6 \mathrm{GHz}$ frequency range: the $K$-structure of the $J=9-8$ transition. The simulated spectrum (in red, top panel) has been obtained from the fit of only four rotational transitions $(J=9 \leftarrow 8 ; K=0,1)$ assigned with the support of the simulated spectrum of Figure 7. The blue-colored experimental spectrum is shown in the bottom panel.

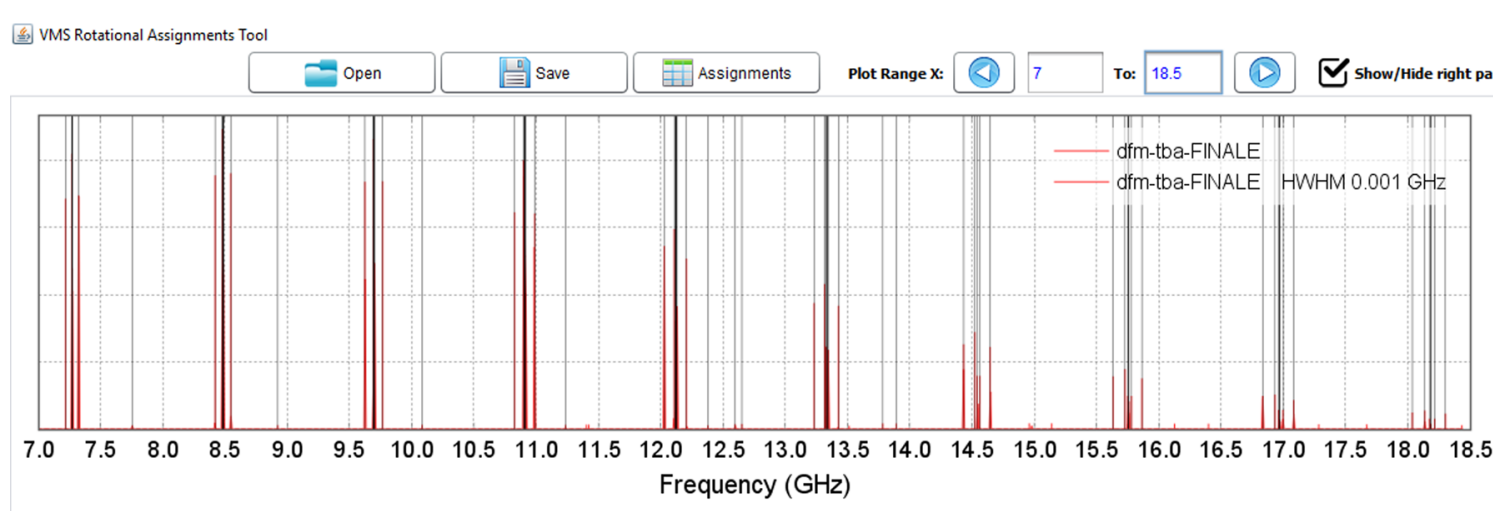

Load an Calculated Spetrum from VCAT Files
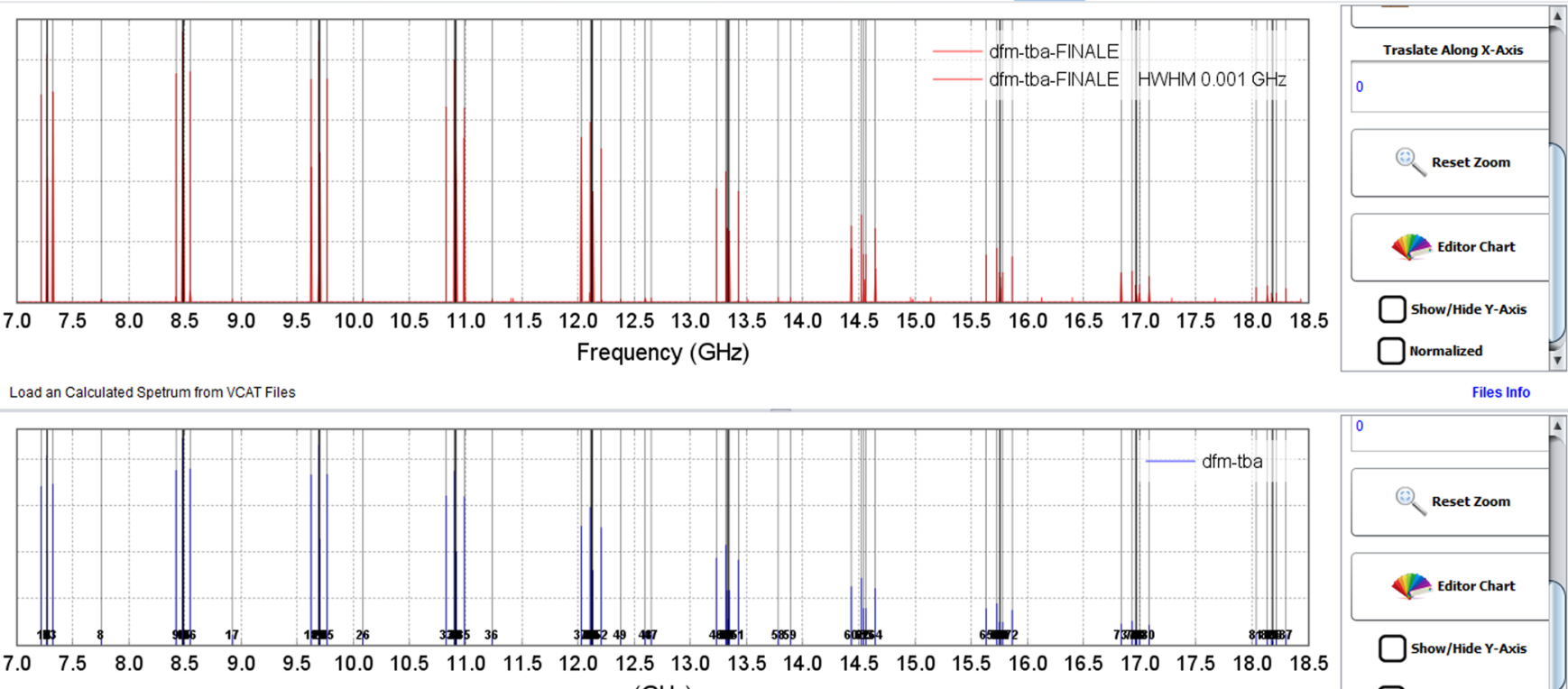

Files Info

$(\mathrm{GHz})$

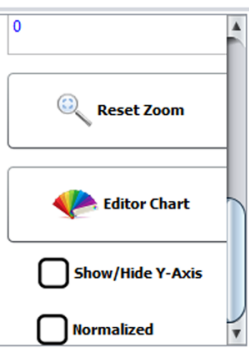

How to Cite...

Open Rotational Simulation performed with VMS Draw (.VCAT File)

Figure 9. VMS-ROT Assignment tool during the analysis of the DFM $\cdots$ TBA complex in the $7.0-18.5 \mathrm{GHz}$ region. Upper panel: simulated spectrum. Lower panel: experimental FTMW spectrum.

software; in fact, it has been employed for studying spectra recorded with different MW spectroscopic techniques, ranging from millimeter-wave spectra to FTMW spectra. The different levels of accuracy of the initial quantum-chemical computations have been checked and validated as well. In addition to being robust and of general use, it should be pointed out that VMS-
ROT (and, in general, VMS-Draw) is also portable on different platforms and operating systems. Additional tests have been also carried out to check the compatibility with the recently released Gaussian 16 version.

VMS-ROT is able to deal with linear, symmetric, and asymmetric rotor spectra, supporting, for the latter, both the $A$ 


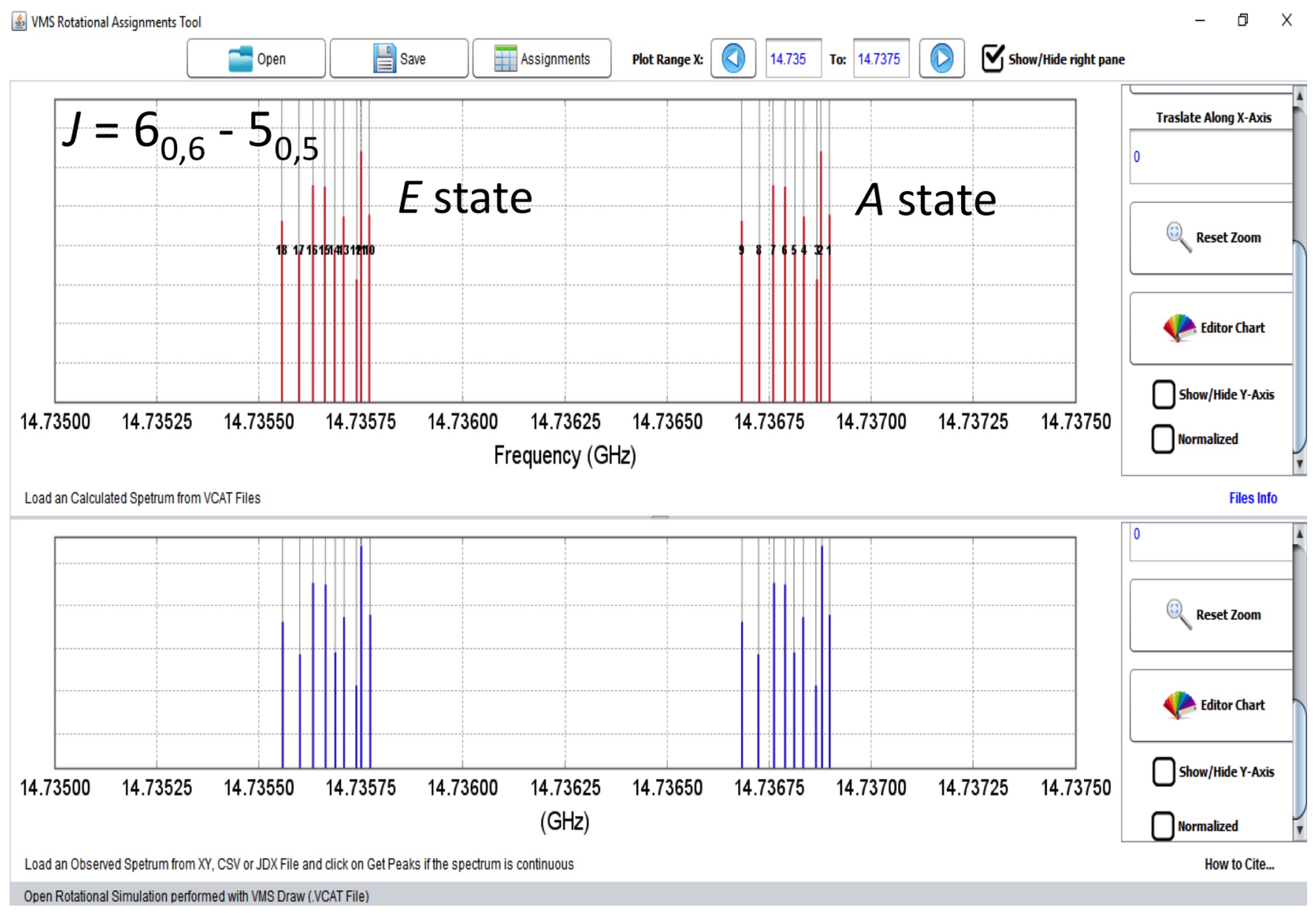

Figure 10. VMS-ROT Assignment tool during the analysis of the PYR $\cdots \mathrm{NH}_{3}$ complex in the 14.7350-14.7375 GHz region: hyperfine structure of the $J$ $=6_{0,6}-5_{0,5}$ rotational transition in the $E$ and $A$ sublevels (for details, see ref 64). Upper panel: simulated spectrum. Lower panel: experimental FTMW spectrum.

and $S$ reductions of Watson's Hamiltonian. Work is in progress to extend it to the proper assignment of complex hyperfine structures arising from the presence of nuclei with nonzero nuclear spin, for which the correct automatic generation of SPFIT and SPCAT input files is not straightforward. Furthermore, the implementation of the automatic assignment and fitting approach, proposed by Seifert et al. ${ }^{15}$ and recently included in the PGOPHER program, ${ }^{18}$ appears straightforward thanks to the object-oriented and modular implementation of VMS-Draw, and it will be shortly implemented. Actually, different strategies for an automatic spectra-assignment prescreening are under investigation. However, although they rely on simple working principles, their black-box implementation, in terms of robustness and general applicability, is not so easy and should be carefully checked.

A final remark concerns the possibility of interfacing VMSROT with spectroscopic databases, such as CDMS or the JPL database, since it can load line listings stored in the .CAT format, while it is a matter of a few lines of code to have the program able to read the transition catalogs according to the HITRAN or GEISA formats. In this context, an appealing further development of VMS-ROT that can be foreseen is its use for the analysis of astronomical observational data. In this case, the relevant observational data, resolved in time and space, can be directly faced with the spectra synthesized from the spectral transitions listed in the above-mentioned databases. The features available for spectral manipulation and for the comparison of more plots at once, not to speak of the possibility of weighting the spectra of different species to generate that of their mixture, makes VMSROT a potential powerful tool for astrophysical applications.

In conclusion, VMS-ROT represents a new useful tool for rotational spectroscopy, which aims at pushing up and automating the interplay between laboratory measurements and computer simulations, one of the most profitable approaches for disclosing the rich information hidden in rotational lines.

\section{APPENDIX A: GETTING AND INSTALLING VMS-ROT}

A beta version of VMS-ROT can be obtained upon request from the authors, while a suitable license policy is currently under examination. The program is written in JAVA, and therefore, it is portable to different platform/operating systems. The minimum system requirement for the current version of VMS-Draw is JAVA 7. After decompressing the VMS archive, the program can be run by executing the VMS.jar file either by mouse-clicking or from the command line typing "java -jar VMS.jar”.

\section{AUTHOR INFORMATION}

\section{Corresponding Author}

*E-mail: vincenzo.barone@sns.it.

ORCID $\odot$

Nicola Tasinato: 0000-0003-1755-7238

Lorenzo Spada: 0000-0003-3273-5303 


\section{Notes}

The authors declare no competing financial interest.

\section{ACKNOWLEDGMENTS}

J. L. Alonso and M. E. Sanz are kindly acknowledged for providing the rotational spectra of methyl- $\beta$-D-xyloside and fenchone, respectively. The SMART@SNS Laboratory (http:// smart.sns.it) is acknowledged for providing high performance computer facilities. The research leading to these results has received funding from the European Research Council under the European Union's Seventh Framework Programme (FP/20072013)/ERC Grant Agreement no. [320951], from MIUR "PRIN 2015" funds (project "STARS in the CAOS (Simulation Tools for Astrochemical Reactivity and Spectroscopy in the Cyberinfrastructure for Astrochemical Organic Species)" Grant Number 2015F59J3R), from Scuola Normale Superiore (Progetti Interni), and from the University of Bologna (RFO funds).

\section{REFERENCES}

(1) Quack, M.; Merkt, F. Handbook of High Resolution Molecular Spectroscopy; John Wiley \& Sons: Chichester, 2011; Vols. 2 and 3.

(2) Puzzarini, C. Computational Strategies for Spectroscopy; Barone, V., Ed.; John Wiley \& Sons: Chichester, 2012; pp 263-307.

(3) Gordy, W.; Cook, L. R. Microwave Molecular Spectra; John Wiley: New York, 1984.

(4) Kroto, H. W. Molecular Rotation Spectra; John Wiley: London, 1975.

(5) Townes, H.; Shawlow, A. L. Microwave Spectroscopy; McGraw-Hill: New York, 1955.

(6) Caminati, W.; Grabow, J.-U. Frontiers of Molecular Spectroscopy; Laane, J., Ed.; Elsevier: Amsterdam, 2009; pp 455-552.

(7) Puzzarini, C.; Stanton, J. F.; Gauss, J. Quantum-chemical calculation of spectroscopic parameters for rotational spectroscopy. Int. Rev. Phys. Chem. 2010, 29, 273-367.

(8) Puzzarini, C. Rotational spectroscopy meets theory. Phys. Chem. Chem. Phys. 2013, 15, 6595-6607.

(9) Shubert, V. A.; Schmitz, D.; Patterson, D.; Doyle, J. M.; Schnell, M. Identifying Enantiomers in Mixtures of Chiral Molecules with Broadband Microwave Spectroscopy. Angew. Chem., Int. Ed. 2014, 53, $1152-1155$.

(10) Abeysekera, C.; Zack, L. N.; Park, G. B.; Joalland, B.; Oldham, J. M.; Prozument, K.; Ariyasingha, N. M.; Sims, I. R.; Field, R. W.; Suits, A. G. A Chirped-pulse Fourier-transform Microwave/Pulsed Uniform Flow Spectrometer II. Performance and Applications to Reaction Dynamics. J. Chem. Phys. 2014, 141, 214203.

(11) Software Packages for Broadband High-Resolution. Spectroscopyhttp://info.ifpan.edu.pl/ kisiel/bband.htm (accessed 19 July 2017).

(12) Western, C. M. PGOPHER: A program for simulating rotational, vibrational and electronic spectra. J. Quant. Spectrosc. Radiat. Transfer 2017, 186, 221-242.

(13) Crabtree, K. N.; Martin-Drumel, M.-A.; Brown, G. B.; Gaster, S. A.; Hall, T. M.; McCarthy, M. C. Microwave spectral taxonomy: A semiautomated combination of chirped-pulse and cavity Fourier-transform microwave spectroscopy. J. Chem. Phys. 2016, 144, 124201.

(14) Martin-Drumel, M.-A.; McCarthy, M. C.; Patterson, D.; McGuire, B. A.; Crabtree, K. N. Automated microwave double resonance spectroscopy: A tool to identify and characterize chemical compounds. J. Chem. Phys. 2016, 144, 124202.

(15) Seifert, N. A.; Finneran, I. A.; Perez, C.; Zaleski, D. P.; Neill, J. L.; Steber, A. L.; Suenram, R. D.; Lesarri, A.; Shipman, S. T.; Pate, B. H. AUTOFIT, an automated fitting tool for broadband rotational spectra, and applications to 1-hexanal. J. Mol. Spectrosc. 2015, 312, 13-21.

(16) Pérez, C.; Krin, A.; Steber, A. L.; López, J. C.; Kisiel, Z.; Schnell, M. Wetting Camphor: Multi-Isotopic Substitution Identifies the Complementary Roles of Hydrogen Bonding and Dispersive Forces. J. Phys. Chem. Lett. 2016, 7, 154-160.
(17) Evangelisti, L.; Perez, C.; Seifert, N. A.; Pate, B. H.; Dehghany, M.; Moazzen-Ahmadi, N.; McKellar, A. R. W. Theory vs. experiment for molecular clusters: Spectra of OCS trimers and tetramers. J. Chem. Phys. 2015, 142, 104309.

(18) Western, C. M.; Billinghurst, B. E. Automatic assignment and fitting of spectra with PGOPHER. Phys. Chem. Chem. Phys. 2017, 19, 10222-10226.

(19) Kisiel, Z.; Pszczolkowski, L.; Medvedev, I. R.; Winnewisser, M.; De Lucia, F. C.; Herbst, E. J. Mol. Spectrosc. 2005, 233, 231-243.

(20) Plusquellic, D. F. JB95 Spectral Fitting Program, v1.02.4; National Institute of Standards and Technology, 2001).

(21) Puzzarini, C.; Biczysko, M.; Barone, V.; Largo, L.; Peña, I.; Cabezas, C.; Alonso, J. L. Accurate characterization of the peptide linkage in the gas phase: A joint quantum-chemical and rotational spectroscopy study of the glycine dipeptide analogue. J. Phys. Chem. Lett. 2014, 5, 534-540.

(22) McCarthy, M. C.; Gottlieb, C. A.; Gupta, H.; Thaddeus, P. Laboratory and Astronomical Identification of the Negative Molecular Ion $\mathrm{C}_{6} \mathrm{H}^{-}$. Astrophys. J. 2006, 652, L141-L144.

(23) Écija, P.; Cocinero, E. J.; Lesarri, A.; Fernández, J. A.; Caminati, W.; Castaño, F. Rotational Spectroscopy of Antipyretics: Conformation, Structure, and Internal Dynamics of phenazone. J. Chem. Phys. 2013, 138,114304 .

(24) Calabrese, C.; Vigorito, A.; Maris, A.; Mariotti, S.; Fathi, P.; Geppert, W. D.; Melandri, S. Millimeter Wave Spectrum of the Weakly Bound Complex $\mathrm{CH}_{2}=\mathrm{CHCN} \cdot \mathrm{H}_{2} \mathrm{O}$ : Structure, Dynamics, and Implications for Astronomical Search. J. Phys. Chem. A 2015, 119, 11674-11682.

(25) McGuire, B. A.; Martin-Drumel, M.-A.; Thorwirth, S.; Brünken, S.; Lattanzi, V.; Neill, J. L.; Spezzano, S.; Yu, Z.; Zaleski, D. P.; Remijan, A. J.; Pate, B. H.; McCarthy, M. C. Molecular Polymorphism: Microwave Spectra, Equilibrium Structures, and an Astronomical Investigation of the HNCS isomeric family. Phys. Chem. Chem. Phys. 2016, 18, 22693-22705.

(26) Zinn, S.; Medcraft, C.; Betz, T.; Schnell, M. High-Resolution Rotational Spectroscopy Study of the Smallest Sugar Dimer: Interplay of Hydrogen Bonds in the Glycolaldehyde Dimer. Angew. Chem. 2016, 128, 6079-6084.

(27) Møller, C.; Plesset, M. S. Note on an Approximation Treatment for Many-Electron Systems. Phys. Rev. 1934, 46, 618-622.

(28) Császár, A. G.; Allen, W. D.; Schaefer, H. F., III. In pursuit of the ab initio limit for conformational energy prototypes. J. Chem. Phys. 1998, 108, 9751-9764.

(29) Montgomery, J. A., Jr.; Frisch, M. J.; Ochterski, J. W.; Petersson, G. A. A complete basis set model chemistry. VI. Use of density functional geometries and frequencies. J. Chem. Phys. 1999, 110, 2822-2827.

(30) Demaison, J.; Margulés, L.; Boggs, J. E. The Equilibrium C-Cl, $\mathrm{C}-\mathrm{Br}$, and $\mathrm{C}-\mathrm{I}$ Bond Lengths from $\mathrm{Ab}$ Initio Calculations, Microwave and Infrared Spectroscopies, and Empirical Correlations. Struct. Chem. 2003, 14, 159-174.

(31) Puzzarini, C. Extrapolation to the Complete Basis Set Limit of Structural Parameters: Comparison of Different Approaches. J. Phys. Chem. A 2009, 113, 14530-14535.

(32) Puzzarini, C.; Barone, V. Extending the molecular size in accurate quantum-chemical calculations: the equilibrium structure and spectroscopic properties of uracil. Phys. Chem. Chem. Phys. 2011, 13, 71897197.

(33) Barone, V.; Biczysko, M.; Bloino, J.; Puzzarini, C. Accurate structure, thermodynamic and spectroscopic parameters from CC and CC/DFT schemes: the challenge of the conformational equilibrium in glycine. Phys. Chem. Chem. Phys. 2013, 15, 10094-10111.

(34) Barone, V.; Biczysko, M.; Bloino, J.; Puzzarini, C. Accurate molecular structures and infrared spectra of trans-2,3-dideuterooxirane, methyloxirane, and trans-2,3-dimethyloxirane. J. Chem. Phys. 2014, 141, 034107.

(35) Puzzarini, C.; Cazzoli, G.; Lopez, J. C.; Alonso, J. L.; Baldacci, A.; Baldan, A.; Stopkowicz, S.; Cheng, L.; Gauss, J. Spectroscopic investigation of fluoroiodomethane, $\mathrm{CH}_{2} \mathrm{FI}$ : Fourier-transform micro- 
wave and millimeter-/submillimeter-wave spectroscopy and quantumchemical calculations. J. Chem. Phys. 2011, 134, 174312.

(36) Puzzarini, C.; Cazzoli, G.; Gauss, J. The rotational spectra of $\mathrm{HD}^{17} \mathrm{O}$ and $\mathrm{D}_{2}{ }^{17} \mathrm{O}$ : Experiment and quantum-chemical calculations. $J$. Chem. Phys. 2012, 137, 154311.

(37) Puzzarini, C.; Biczysko, M.; Barone, V.; Peña, I.; Cabezas, C.; Alonso, J. L. Accurate molecular structure and spectroscopic properties of nucleobases: a combined computational-microwave investigation of 2-thiouracil as a case study. Phys. Chem. Chem. Phys. 2013, 15, 1696516975.

(38) McCarthy, M. C.; Gauss, J. Exotic $\mathrm{SiO}_{2} \mathrm{H}_{2}$ Isomers: Theory and Experiment Working in Harmony. J. Phys. Chem. Lett. 2016, 7, 18951900.

(39) Spada, L.; Tasinato, N.; Vazart, F.; Barone, V.; Caminati, W.; Puzzarini, C. Noncovalent Interactions and Internal Dynamics in Pyridine-Ammonia: A Combined Quantum-Chemical and Microwave Spectroscopy Study. Chem. - Eur. J. 2017, 23, 4876-4883.

(40) Licari, D.; Baiardi, A.; Biczysko, M.; Egidi, F.; Latouche, C.; Barone, V. Implementation of a Graphical User Interface for the Virtual Multifrequency Spectrometer: The VMS-Draw Tool. J. Comput. Chem. 2015, 36, 321-334.

(41) Barone, V. The Virtual Multifrequency Spectrometer: a New Paradigm for Spectroscopy. WIREs Comput.Mol.Sci. 2016, 6, 86-110.

(42) Frisch, M. J.; Trucks, G. W.; Schlegel, H. B.; Scuseria, G. E.; Robb, M. A.; Cheeseman, J. R.; Scalmani, G.; Barone, V.; Petersson, G. A.; Nakatsuji, H.; Li, X.; Caricato, M.; Marenich, A. V.; Bloino, J.; Janesko, B. G.; Gomperts, R.; Mennucci, B.; Hratchian, H. P.; Ortiz, J. V.; Izmaylov, A. F.; Sonnenberg, J. L.; Williams-Young, D.; Ding, F.; Lipparini, F.; Egidi, F.; Goings, J.; Peng, B.; Petrone, A.; Henderson, T.; Ranasinghe, D.; Zakrzewski, V. G.; Gao, J.; Rega, N.; Zheng, G.; Liang, W.; Hada, M.; Ehara, M.; Toyota, K.; Fukuda, R.; Hasegawa, J.; Ishida, M.; Nakajima, T.; Honda, Y.; Kitao, O.; Nakai, H.; Vreven, T.; Throssell, K.; Montgomery, J. A., Jr.; Peralta, J. E.; Ogliaro, F.; Bearpark, M. J.; Heyd, J. J.; Brothers, E. N.; Kudin, K. N.; Staroverov, V. N.; Keith, T. A.; Kobayashi, R.; Normand, J.; Raghavachari, K.; Rendell, A. P.; Burant, J. C.; Iyengar, S. S.; Tomasi, J.; Cossi, M.; Millam, J. M.; Klene, M.; Adamo, C.; Cammi, R.; Ochterski, J. W.; Martin, R. L.; Morokuma, K.; Farkas, O.; Foresman, J. B.; Fox, D. J. Gaussian 16, Revision A.03, Gaussian, Inc.: Wallingford, CT, 2016.

(43) Zerbetto, M.; Licari, D.; Barone, V.; Polimeno, A. Computational Tools for the Interpretation of Electron Spectra in Solution. Mol. Phys. 2013, 111, 2746-2756.

(44) Presti, D.; Pedone, A.; Licari, D.; Barone, V. A Modular Implementation for the Simulation of $1 \mathrm{D}$ and 2D Solid-State NMR Spectra of Quadrupolar Nuclei in the Virtual Multifrequency Spectrometer-Draw Graphical Interface. J. Chem. Theory Comput. 2017, 13, 2215-2229.

(45) Mendolicchio, M.; Penocchio, E.; Licari, D.; Tasinato, N.; Barone, V. Development and Implementation of Advanced Fitting Methods for the Calculation of Accurate Molecular Structures. J. Chem. Theory Comput. 2017, 13, 3060.

(46) Penocchio, E.; Mendolicchio, M.; Tasinato, N.; Barone, V. Structural features of the carbon-sulfur chemical bond: a semiexperimental perspective. Can. J. Chem. 2016, 94, 1065-1076.

(47) Pickett, H. M. The fitting and prediction of vibration-rotation spectra with spin interactions. J. Mol. Spectrosc. 1991, 148, 371-377.

(48) Tasinato, N.; Stoppa, P.; Pietropolli Charmet, A.; Giorgianni, S.; Gambi, A. High resolution infrared study of vinyl fluoride in the $750-$ $1050 \mathrm{~cm}^{-1}$ region: rovibrational analysis and resonances involving the $\nu_{8}, \nu_{10}$ and $\nu_{11}$ fundamentals. J. Phys. Chem. A 2006, 110, 13412-13418.

(49) Aviles-Moreno, J.-R; Demaison, J.; Huet, T. R. Conformational Flexibility in Hydrated Sugars: The Glycolaldehyde-Water Complex. J. Am. Chem. Soc. 2006, 128, 10467-10473.

(50) Carroll, P. B.; McGuire, B. A.; Zaleski, D. P.; Neill, J. L.; Pate, B. H.; Widicus Weaver, S. L. The Pure Rotational Spectrum of Glycolaldehyde Isotopologues Observed in Natural Abundance. J. Mol. Spectrosc. 2013, 284-285, 21-28.

(51) Schmitz, D.; Shubert, V. A.; Giuliano, B. M.; Schnell, M. The Broadband Microwave Spectra of the Monoterpenoids Thymol and
Carvacrol: Conformational Landscape and Internal Dynamics. J. Chem. Phys. 2014, 141, 034304.

(52) Zou, L.; Hays, B. M.; Widicus Weaver, S. L. Weakly Bound Clusters in Astrochemistry? Millimeter and Submillimeter Spectroscopy of trans-HO3 and Comparison to Astronomical Observations. J. Phys. Chem. A 2016, 120, 657-667.

(53) Dorosh, O.; Białkowska-Jaworska, E.; Kisiel, Z.; Pszczółkowski, L.; Kańska, M.; Krygowski, T. M.; Mäder, H. The complete molecular geometry and electric dipole moment of salicyl aldehyde from rotational spectroscopy. J. Mol. Spectrosc. 2017, 335, 3-12.

(54) Müller, H. S. P.; Schlöder, F.; Stutzki, J.; Winnewisser, G. The Cologne Database for Molecular Spectroscopy, CDMS: a Useful Tool for Astronomers and Spectroscopists. J. Mol. Struct. 2005, 742, 215227.

(55) Pickett, H. M.; Poynter, R. L.; Cohen, E. A.; Delitsky, M. L.; Pearson, J. C.; Muller, H. S. P. Submillimeter, millimeter, and microwave spectral line catalogue. J. Quant. Spectrosc. Radiat. Transfer 1998, 60, 883-890.

(56) CFOUR, a quantum chemical program package written by Stanton, J.F.; Gauss, J.; Harding, M.E.; Szalay, P.G., with contributions from Auer, A.A.; Bartlett, R.J.; Benedikt, U.; Berger, C.; Bernholdt, D.E.; Bomble, Y.J.; Cheng, L.; Christiansen, O.; Engel, F.; Faber, R.; Heckert, M.; Heun, O.; Huber, C.; Jagau, T.-C.; Jonsson, D.; Jusélius, J., Klein, K.; Lauderdale, W.J.; Lipparini, F.; Matthews, D.A.; Metzroth, T.; Mück, L. A., O’Neill, D. P., Price, D.R.; Prochnow, E.; Puzzarini, C.; Ruud, K.; Schiffmann, F.; Schwalbach, W.; Simmons, C.; Stopkowicz, S.; Tajti, A.; Vázquez, J.; Wang, F.; Watts, J. D. and the integral packages MOLECULE (Almlöf, J.; Taylor, P.R.), PROPS (Taylor, P.R.), ABACUS (Helgaker, T.; Jensen, H. J. A., Jørgensen, P.; Olsen, J.), and ECP routines by Mitin, A. V.; van Wüllen, C.. For the current version, see http://www.cfour.de.

(57) Spada, L.; Gou, Q.; Vallejo-López, M.; Lesarri, A.; Cocinero, E. J.; Caminati, W. Weak $\mathrm{C}-\mathrm{H} \cdots \mathrm{N}$ and $\mathrm{C}-\mathrm{H} \cdots \mathrm{F}$ hydrogen bonds and internal rotation in pyridine- $\mathrm{CH}_{3}$ F. Phys. Chem. Chem. Phys. 2014, 16, 2149-2153.

(58) Evangelisti, L.; Spada, L.; Li, W.; Blanco, S.; López, J. C.; Lesarri, A.; Grabow, J.-U.; Caminati, W. A butterfly motion of formic acid and cyclobutanone in the 1:1 hydrogen bonded molecular cluster. Phys. Chem. Chem. Phys. 2017, 19, 204-209.

(59) Papoušek, D.; Aliev, M. R. Molecular Vibrational/Rotational Spectra; Elsevier: Amsterdam, 1982.

(60) Aliev, M. R.; Watson, J. K. G. Molecular Spectroscopy: Modern Research, Vol. 3; Rao, K. N., Ed.; Academic Press, Inc.: Orlando, FL, 1985; pp 1-67.

(61) Pietropolli Charmet, A.; Stoppa, P.; Giorgianni, S.; Bloino, J.; Tasinato, N.; Carnimeo, I.; Biczysko, M.; Puzzarini, C. Accurate Vibrational-Rotational Parameters and Infrared Intensities of 1Bromo-1-fluoroethene: A Joint Experimental Analysis and $\mathrm{Ab}$ Initio Study. J. Phys. Chem. A 2017, 121, 3305-3317.

(62) Purvis, G. D., III; Bartlett, R. J. A full coupled-cluster singles and doubles model: The inclusion of disconnected triples. J. Chem. Phys. 1982, 76, 1910-1918.

(63) Raghavachari, K.; Trucks, G. W.; Pople, J. A.; Head-Gordon, M. A Fifth-Order Perturbation Comparison of Electron Correlation Theories. Chem. Phys. Lett. 1989, 157, 479-483.

(64) Spada, L.; Tasinato, N.; Bosi, G.; Vazart, F.; Barone, V.; Puzzarini, C. On the competition between weak $\mathrm{O}-\mathrm{H} \cdots \mathrm{F}$ and $\mathrm{C}-\mathrm{H} \cdots \mathrm{F}$ hydrogen bonds, in cooperation with $\mathrm{C}-\mathrm{H} \cdots \mathrm{O}$ contacts, in the difluoromethane tert-butyl alcohol cluster. J. Mol. Spectrosc. 2017, 337, 90-95.

(65) McQuire, D. A.; Simon, J. D. Molecular Thermodynamics; University Science Books: Sausalito, CA, 1999.

(66) Tasinato, N.; Pietropolli Charmet, A.; Stoppa, P. ATIRS package: A program suite for the rovibrational analysis of infrared spectra of asymmetric top molecules. J. Mol. Spectrosc. 2007, 243, 148-154.

(67) Heineking, N.; Dreizler, H.; Schwarz, R. Nitrogen and Deuterium Hyperfine Structure in the Rotational Spectra of Pyridine and [4-D] Pyridine. Z. Naturforsch., A: Phys. Sci. 1986, 41, 1210-1213.

(68) Puzzarini, C. Accurate molecular structures of small- and mediumsized molecules. Int. J. Quantum Chem. 2016, 116, 1513-1519. 
(69) Grimme, S. Semiempirical Hybrid Density Functional with Perturbative Second-Order Correlation. J. Chem. Phys. 2006, 124, 034108.

(70) Papajak, E.; Leverentz, H. R.; Zheng, J.; Truhlar, D. G. Efficient Diffuse Basis Sets: cc-pVxZ+ and maug-cc-pVxZ. J. Chem. Theory Comput. 2009, 5, 1197-1202.

(71) Grimme, S.; Antony, J.; Ehrlich, S.; Krieg, H. A Consistent and Accurate $a b$ initio Parametrization of Density Functional Dispersion Correction (DFT-D) for the 94 Elements H-Pu. J. Chem. Phys. 2010, 132, 154104.

(72) Grimme, S.; Ehrlich, S.; Goerigk, L. Effect of the damping function in dispersion corrected density functional theory. J. Comput. Chem. 2011, 32, 1456-1465.

(73) Loru, D.; Bermúdez, M. A.; Sanz, M. E. Structure of Fenchone by Broadband Rotational Spectroscopy. J. Chem. Phys. 2016, 145, 074311.

(74) Krishnan, R.; Binkley, J. S.; Seeger, R.; Pople, J. A. Self-consistent molecular orbital methods. XX. A basis set for correlated wave functions. J. Chem. Phys. 1980, 72, 650-654.

(75) Watson, J. K. G. Vibrational Spectra and Structure, Vol. 6; During, J. R., Ed.; Elsevier: New York, p1977; p 1-80.

(76) Alonso, E. R.; Peña, I.; Cabezas, C.; Alonso, J. L. Structural Expression of Exo-Anomeric Effect. J. Phys. Chem. Lett. 2016, 7, 845850.

(77) he Cologne Database for Molecular Spectroscopy. https://www. astro.uni-koeln.de/cdms (accessed 18 May 2017).

(78) Cazzoli, G.; Puzzarini, C. The Lamb-dip spectrum of methylcyanide: Precise rotational transition frequencies and improved ground-state rotational parameters. J. Mol. Spectrosc. 2006, 240, 153163.

(79) Cazzoli, G.; Puzzarini, C. Corrigendum to "The Lamb-dip spectrum of methylcyanide: Precise rotational transition frequencies and improved ground-state rotational parameters" [J. Mol. Spectrosc. 240 (2006) 153-163]. J. Mol. Spectrosc. 2008, 247, 187-188.

(80) Rothman, L. S.; Gordon, I. E.; Babikov, Y.; Barbe, A.; Chris Benner, D.; Bernath, P. F.; Birk, M.; Bizzocchi, L.; Boudon, V.; Brown, L. R.; Campargue, A.; Chance, K.; Cohen, E. A.; Coudert, L. H.; Devi, V. M.; Drouin, B. J.; Fayt, A.; Flaud, J.-M.; Gamache, R. R.; Harrison, J. J.; Hartmann, J.-M.; Hill, C.; Hodges, J. T.; Jacquemart, D.; Jolly, A.; Lamouroux, J.; Le Roy, R. J.; Li, G.; Long, D. A.; Lyulin, O. M.; Mackie, C. J.; Massie, S. T.; Mikhailenko, S.; Müller, H. S. P.; Naumenko, O. V.; Nikitin, A. V.; Orphal, J.; Perevalov, V.; Perrin, A.; Polovtseva, E. R.; Richard, C.; Smith, M. A. H.; Starikova, E.; Sung, K.; Tashkun, S.; Tennyson, J.; Toon, G. C.; Tyuterev, V.; Wagner, G. The HITRAN2012 Molecular Spectroscopic Database. J. Quant. Spectrosc. Radiat. Transfer 2013, 130, 4-50.

(81) Jacquinet-Husson, N.; Armante, R.; Scott, N. A.; Chédin, A.; Crépeau, L.; Boutammine, C.; Bouhdaoui, A.; Crevoisier, C.; Capelle, V.; Boonne, C.; Poulet-Crovisier, N.; Barbe, A.; Chris Benner, D.; Boudon, V.; Brown, L. R.; Buldyreva, J.; Campargue, A.; Coudert, L. H.; Devi, V. M.; Down, M. J.; Drouin, B. J.; Fayt, A.; Fittschen, C.; Flaud, J.M.; Gamache, R. R.; Harrison, J. J.; Hill, C.; Hodnebrog, Ø.; Hu, S.-M.; Jacquemart, D.; Jolly, A.; Jiménez, E.; Lavrentieva, N. N.; Liu, A.-W.; Lodi, L.; Lyulin, O. M.; Massie, S. T.; Mikhailenko, S.; Müller, H. S. P.; Naumenko, O. V.; Nikitin, A.; Nielsen, C. J.; Orphal, J.; Perevalov, V. I.; Perrin, A.; Polovtseva, E.; Predoi-Cross, A.; Rotger, M.; Ruth, A. A.; Yu, S. S.; Sung, K.; Tashkun, S. A.; Tennyson, J.; Tyuterev, V. G.; Vander Auwera, J.; Voronin, B. A.; Makie, A. The 2015 Edition of the GEISA Spectroscopic Database. J. Mol. Spectrosc. 2016, 327, 31-72.

(82) Puzzarini, C.; Cazzoli, G. Equilibrium structure of methylcyanide. J. Mol. Spectrosc. 2006, 240, 260-264. 